\title{
HARD-CORE THINNINGS OF GERM-GRAIN MODELS WITH POWER-LAW GRAIN SIZES
}

\author{
MIKKO KURONEN *** AND \\ LASSE LESKELÄ, ${ }^{* * * *}$ University of Jyväskylä
}

\begin{abstract}
Random sets with long-range dependence can be generated using a Boolean model with power-law grain sizes. We study thinnings of such Boolean models which have the hardcore property that no grains overlap in the resulting germ-grain model. A fundamental question is whether long-range dependence is preserved under such thinnings. To answer this question, we study four natural thinnings of a Poisson germ-grain model where the grains are spheres with a regularly varying size distribution. We show that a thinning which favors large grains preserves the slow correlation decay of the original model, whereas a thinning which favors small grains does not. Our most interesting finding concerns the case where only disjoint grains are retained, which corresponds to the wellknown Matérn type-I thinning. In the resulting germ-grain model, typical grains have exponentially small sizes, but rather surprisingly, the long-range dependence property is still present. As a byproduct, we obtain new mechanisms for generating homogeneous and isotropic random point configurations having a power-law correlation decay.
\end{abstract}

Keywords: Regular variation; Boolean model; marked Poisson process; germ-grain model; hard-core model; hard-sphere model

2010 Mathematics Subject Classification: Primary 60D05

Secondary 60G55

\section{Introduction}

Consider a random closed set which can be expressed as a union of compact sets in the $d$-dimensional Euclidean space $\mathbb{R}^{d}$. The compact building blocks of the random set are called grains. This kind of random object is often called the germ-grain model. Our focus is on models with the hard-core property, that is, the grains are disjoint with probability 1 . This kind of model (also known as random packing models) provides an important class of mathematical tools for the natural sciences, allowing us to model and analyze the statistical features of disordered porous materials [18], [21]. Besides natural sciences, these models have found applications in engineering when analyzing the performance of medium access protocols in wireless data networks (see, e.g. [2], [10], and [17]).

A key statistical feature of a random set is its covariance function, which describes how much more or less likely it is to find matter at a given distance from a location containing matter, compared to finding matter in an arbitrary location. While most germ-grain models studied in the literature have a rapidly decaying covariance function, certain experimental studies in astronomy [11] and materials science [21] display real-world data where the statistically

Received 17 April 2012; revision received 8 October 2012.

* Postal address: Department of Mathematics and Statistics, University of Jyväskylä, PO Box 35, Jyväskylä 40014, Finland.

** Email address: mikko.p.o.kuronen@jyu.fi

*** Email address: lasse.leskela@iki.fi 
estimated covariance function appears to decay exceptionally slowly, following a power law $r^{-\beta}$ with some exponent $\beta>0$ for large distances $r$. When $\beta<d$, such models are long-range dependent in the sense that

$$
\limsup _{r \rightarrow \infty} \frac{\operatorname{var}\left(\left|X \cap B_{r}\right|\right)}{r^{d}}=\infty,
$$

where $\left|X \cap B_{r}\right|$ denotes the volume of the region covered by the random set $X$ within the closed ball $B_{r}$ with radius $r$ centered at the origin [7, Section 12.7]. Long-range dependence causes anomalous behavior to several statistical features of the model, as is well understood in time series analysis [19]. Note that, for a homogeneous random set in dimension $d=1$, property (1.1) is equivalent to the usual notion of long-range dependence,

$$
\limsup _{n \rightarrow \infty} \frac{\operatorname{var}\left(\sum_{k=1}^{n} X_{k}\right)}{n}=\infty,
$$

of the time series $X_{k}=|X \cap(k-1, k]|$.

Our goal in this paper is to construct parsimonious germ-grain models having the hardcore and long-range dependence property. In the presence of long-range dependence, the requirement of parsimony, i.e. having a small number of model parameters, is especially important because long-range dependence tends to reduce the robustness of the statistical estimators of model parameters [5]. Long-range dependent germ-grain models are easy to generate using a Boolean model - a germ-grain model with random power-law distributed sizes and independently and uniformly scattered centers-but the resulting model is not hardcore by construction. To make it hard core, we will follow Matérn's approach [15] of thinning out a selected collection of overlapping grains from the proposed Boolean model so that the resulting collection of grains is disjoint. Whether this approach is feasible for obtaining hardcore models with long-range dependence depends on the following question.

Is the power-law covariance decay of the proposed Boolean model preserved after making it disjoint by thinning?

To answer this question, we analyze in detail the following natural thinning mechanisms.

- Large retained. Let the thinned model consist of those grains in the original Boolean model which are not overlapped by any larger grain in the original model.

- Random retained. Assign independent random weights to the grains. Let the thinned model consist of those grains in the original model which are not overlapped by any heavier grain in the original model. (This thinning corresponds to Matérn type II.)

- Small retained. Let the thinned model consist of those grains in the original model which are not overlapped by any smaller grain in the original model.

- Isolated retained. Let the thinned model be the set of grains in the original model which do not overlap with any other grain in the original model. (This thinning corresponds to Matérn type I.)

We remark that—unlike the Matérn type-III hard-core model [17]— the above thinnings are local in that the decision whether a proposed grain will be retained or not is made solely by looking at the grains which intersect it.

For simplicity, we will restrict to spherical models where the grains are closed balls. In Figure 1 we illustrate the above four thinnings applied to a simulated sample of a Boolean 

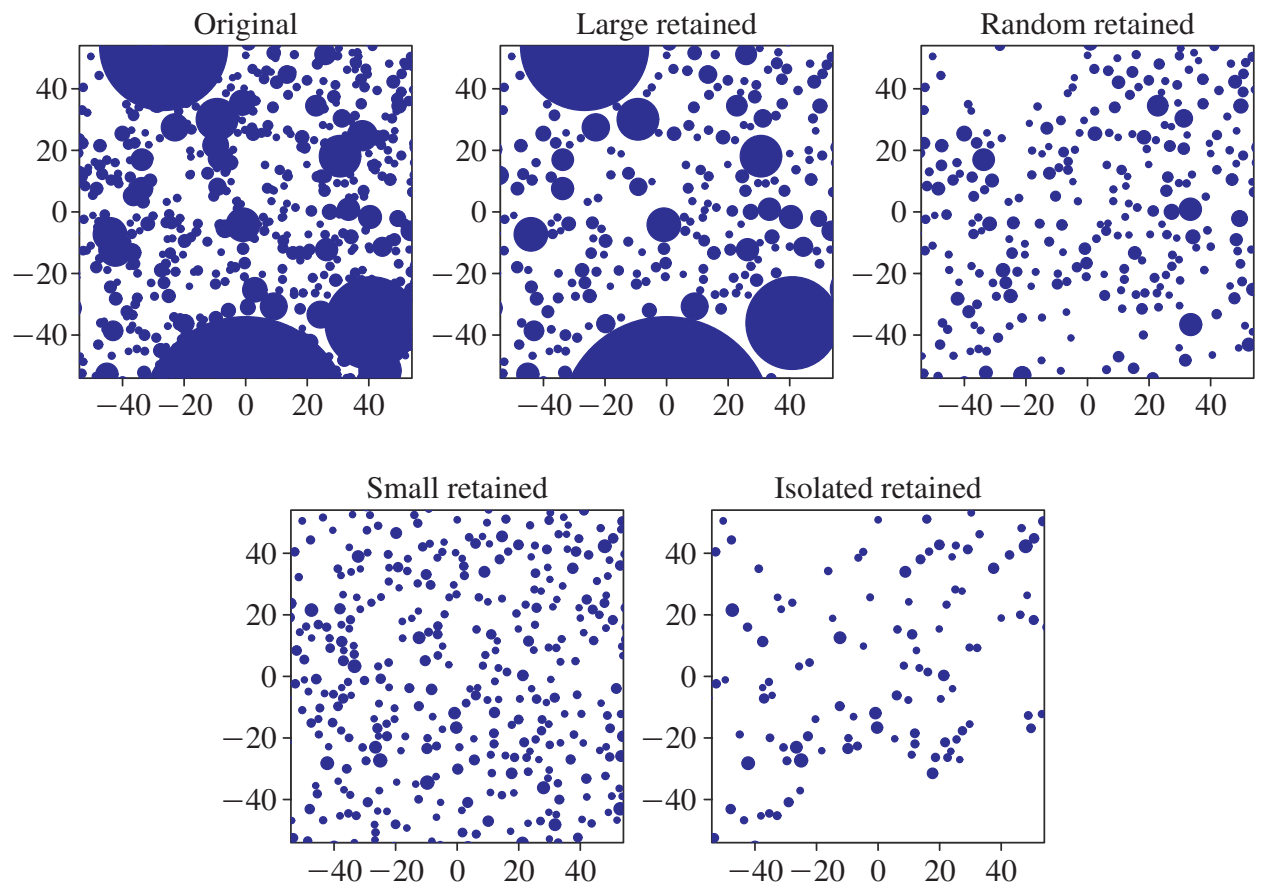

FIGURE 1: Original model and its four hard-core thinnings, where the original model is generated by disks having a Pareto distribution with tail exponent $\alpha=2.5$.

model in $\mathbb{R}^{2}$ where the grain centers have mean density $\lambda=0.05$ and the grain radii have a Pareto distribution $F(r)=1-r^{-\alpha}, r \geq 1$, with tail exponent $\alpha=2.5$.

The above thinnings will be analyzed collectively by viewing them as instances of a general weight-based thinning mechanism, following Månsson and Rudemo [14]. Standard formulae of Palm calculus allow us to write down closed-form analytical formulae for the radius distribution of a typical grain, the covariance function of the grain union, and the two-point correlation function of the grain centers for general hard-core germ-grain configurations generated by weight-based thinnings. Using the theory of regular variation, we analyze the long-range behavior of these quantities under the assumption that the grain radii in the proposed Boolean model follow a power-law distribution with tail exponent $\alpha>d$.

The main results of this paper (Theorems 5.1, 6.1, 7.1, 8.1) are summarized in Table 1. From the table, we can draw the following conclusions:

- The power-law covariance decay and long-range dependence (when $\alpha<2 d$ ) of the grain union are preserved under all thinnings except small retained.

- Whereas the random point configuration (also known as the point process) of grain centers in the proposed Boolean model is completely uncorrelated, the corresponding point configurations in all thinned models except small retained have a power-law twopoint correlation function.

- The heavy tail of the grain radius distribution is destroyed by small retained and isolated retained thinnings. The other two thinnings preserve the power-law structure of the tail distribution: under large retained with the same exponent, under random retained with a larger exponent corresponding to a lighter tail. 
TABLE 1: Long-range decay of key statistical characteristics of the original Boolean model and the hardcore germ-grain models obtained by thinning.

\begin{tabular}{llll}
\hline \multicolumn{1}{c}{ Model } & $\begin{array}{c}\text { Radius distribution } \\
\text { of a typical grain }\end{array}$ & $\begin{array}{c}\text { Covariance function } \\
\text { of grain union }\end{array}$ & $\begin{array}{c}\text { Correlation function } \\
\text { of grain centers }\end{array}$ \\
\hline Original & Power law $(\alpha)$ & Power law $(\alpha-d)$ & Zero \\
Large retained & Power law $(\alpha)$ & Power law $(\alpha-d)$ & Power law $(\alpha-d)$ \\
Random retained & Power law $(\alpha+d)$ & Power law $(\alpha-d)$ & Power law $(\alpha-d)$ \\
Small retained & Exponential & Exponential & Exponential \\
Isolated retained & Exponential & Power law $(\alpha-d)$ & Power law $(\alpha-d)$ \\
\hline
\end{tabular}

Table 1 also reveals a striking feature of the isolated retained thinning mechanism: the resulting random set and the resulting point configuration of grain centers both exhibit long-range dependence although the grain size distribution is light tailed. This seemingly paradoxical phenomenon can be explained by inspecting the empty space. Any region of space not covered by the grains of the thinned model is likely to have been contained in a big grain of the proposed model that was removed in the thinning, and, therefore, a large neighborhood of this empty region is likely to be empty, too.

This paper may be seen as a continuation of the works of Månsson and Rudemo [14] and Andersson et al. [1], who analyzed first-order statistical properties of hard-core germ-grain models obtained by weight-based thinnings. In [14, Corollary 3.1] it was also shown that large retained thinning preserves the tail behavior of the typical grain radius whenever the proposed grain radius distribution is continuous. A slightly more general thinning framework was recently introduced by Nguyen and Baccelli [17], who derived differential equations characterizing the generating functional of the random point configuration formed by the thinned grain centers. Earlier work on the covariance analysis of random sets includes Böhm and Schmidt [4], who derived a short-range approximation for the covariance function of a general homogeneous random set. Snethlage et al. [22] (see also the references therein) provided a nice summary of random point configuration models where the two-point correlation function has a powerlaw behavior on short distances. Earlier works on long-range-dependent random sets appear mostly restricted to random point configurations in dimension $d=1$. Among these, Daley and Vesilo [8] established the following elegant preservation property for many queueing systems: the point configuration of the departure times is long-range dependent if and only if the same is true for the arrival times. Daley [6] showed that a renewal point process is long-range dependent if the interpoint distances have an infinite second moment, and Kulik and Szekli [13] extended this observation to one-dimensional point configurations with positively associated interpoint distances. Vamvakos and Anantharam [24] showed that the long-range dependence of a point process is preserved by a leaky bucket flow control mechanism for data traffic. A study focused on the long-range dependence of multidimensional random sets is the recent work of Demichel et al. [9], who studied whether random sets having power-law decaying chord length distributions, closely related to the covariance function of the random set, can be generated as a level set of a Gaussian random field - they found that in wide generality (merely assuming that the underlying Gaussian field is mixing), this is not possible.

Let us summarize the notational conventions used in this paper. The symbol $\mathrm{P}$ stands for the probability measure on some abstract probability space which governs all randomness in the models, and E, var, and cov denote the expectation, variance, and covariance with respect to $\mathrm{P}$, respectively. The symbol $B_{r}(x)$ denotes the closed unit ball with center $x$ and radius $r$ 
in the $d$-dimensional Euclidean space $\mathbb{R}^{d}$. We use $B_{r}$ as shorthand for $B_{r}(o)$, where $o$ is the origin of $\mathbb{R}^{d}$. For a Borel set $B$ in $\mathbb{R}^{d}$, we denote by $|B|$ its Lebesgue measure, and by $\mathbf{1}_{B}(x)$ or $\mathbf{1}(x \in B)$ its indicator function. The symbols $\mathrm{d} x, \mathrm{~d} y$, etc. refer to the Lebesgue measure in $\mathbb{R}^{d}$. The symbol $\mathbb{R}_{+}$denotes the positive real numbers including 0 . The symbol $F(\mathrm{~d} r)$ refers to integration with respect to a probability measure $F$ on $\mathbb{R}_{+}$, whereas $F(r)=F[0, r]$ and $\bar{F}(r)=1-F(r)$ stand for the corresponding cumulative distribution function and the complementary cumulative distribution function, respectively. The minimum and maximum of real numbers $a$ and $b$ are denoted by $a \wedge b$ and $a \vee b$, respectively. When convenient, we write $\int_{a}^{\infty}=\int_{(a, \infty)}, \int_{0}^{b}=\int_{[0, b]}$, and $\int_{a}^{b}=\int_{(a, b]}$ for $0<a<b<\infty$. For functions $f$ and $g$ defined on the positive real line, we write $f \sim g$ if $f(t) / g(t) \rightarrow 1$ as $t \rightarrow \infty$.

The rest of the paper is organized as follows. In Section 2 we summarize preliminaries on random Boolean models needed later in the text. In Section 3 we introduce a weight-based thinning mechanism which produces hard-core germ-grain models from Boolean models and list formulae for the second-order characteristics of the models so obtained. Section 4 contains a long-range analysis of the second-order characteristics of the previous section. The main results of Table 1 are proved case-by-case in Section 5 (isolated retained), Section 6 (random retained), Section 7 (large retained), and Section 8 (small retained). Section 9 concludes the paper.

\section{Boolean models with power-law grain radii}

We consider a random collection of closed spheres, where the sphere centers are independently and uniformly scattered in $\mathbb{R}^{d}$ and the sphere radii are independent and identically distributed random variables in $\mathbb{R}_{+}$. Mathematically, this model can be defined using a Poisson process $\Phi$ on $\mathbb{R}^{d} \times \mathbb{R}_{+}$with intensity measure $\lambda \mathrm{d} x F(\mathrm{~d} r)$, where $\lambda$ is a positive constant and $F$ is a probability measure on $\mathbb{R}_{+}$such that $\int r^{d} F(\mathrm{~d} r)<\infty$. We identify each pair $(x, r) \in \Phi$ with the closed ball $B_{r}(x)$ with center $x$ and radius $r$ and-conforming to the terminology of more general germ-grain models-such pairs will be called grains. We denote by

$$
X=\bigcup_{(x, r) \in \Phi} B_{r}(x)
$$

the grain union of $\Phi$, and by

$$
\Phi_{g}=\left\{x \in \mathbb{R}^{d}:(x, r) \in \Phi \text { for some } r\right\}
$$

the random point configuration in $\mathbb{R}^{d}$ formed by the grain centers of $\Phi$. Note that $\Phi_{g}$ is a homogeneous Poisson process on $\mathbb{R}^{d}$ with intensity $\lambda$. The parameter $\lambda$ thus equals the mean density of grain centers, and the probability measure $F$ is the common distribution of grain radii. For general definitions and details about random sets and point processes, see, for example, [7], [16], [20], and [23].

The covariance of the random set $X$ is denoted by $k(x, y)=\operatorname{cov}\left(\mathbf{1}_{X}(x), \mathbf{1}_{X}(y)\right)$, where $\mathbf{1}_{X}$ is the indicator function of $X$. Because the distribution of $X$ is shift invariant by construction, the covariance is given by $k(x, y)=k(x-y)$, where the covariance function $k(z)=k(o, z)$ is given by the well-known formula (see, e.g. [23, Section 3.1])

$$
k(z)=(1-p)^{2}\left(\exp \left(\lambda \int\left|B_{r}(o) \cap B_{r}(z)\right| F(\mathrm{~d} r)\right)-1\right),
$$


and where $p$ is the volume fraction of $X$ given by

$$
p=1-\exp \left(-\lambda \int\left|B_{r}\right| F(\mathrm{~d} r)\right) .
$$

Formula (2.1) indeed shows that $k(z)$ depends on $z$ only through $|z|$, which is evident because $X$ is isotropic by construction. Using this formula we may also deduce that

$$
k(z) \sim \lambda(1-p)^{2} \int\left|B_{r}(o) \cap B_{r}(z)\right| F(\mathrm{~d} r) \quad \text { as }|z| \rightarrow \infty,
$$

where we write $f(z) \sim g(z)$ if $f(z) / g(z) \rightarrow 1$ as $|z| \rightarrow \infty$. When the grain radius distribution $F$ follows a power law with tail exponent $\alpha>d$, so that $F(r):=F[0, r]=1-\ell(r) r^{-\alpha}$ for some slowly varying function $\ell$ (see Appendix A for details), it follows by using Lemma B.1 in Appendix B that

$$
k(z) \sim \lambda(1-p)^{2} c_{\alpha, d} \ell(|z|)|z|^{-(\alpha-d)} \quad \text { as }|z| \rightarrow \infty .
$$

Thus, when the radius distribution follows a power law with tail exponent $\alpha>d$, then the covariance function $k(z)$ follows a power law with tail exponent $\alpha-d$. Especially, the grain union $X$ of Boolean model is long-range dependent in the sense of (1.1) for $\alpha \in(d, 2 d)$.

\section{Weight-based thinning}

In this section we will study a weight-based thinning mechanism which maps a Boolean model into a hard-core germ-grain model consisting of nonoverlapping grains [14], [17]. This thinning mechanism is defined by assigning random weights to the grains of the Boolean model, and retaining those grains which are not overlapped by any other grain in the Boolean model with a higher or equal weight.

\subsection{Thinning mechanism}

A weighted spherical Boolean model is generated by a Poisson process $\Phi$ on $\mathbb{R}^{d} \times \mathbb{R}_{+} \times \mathbb{R}_{+}$ with intensity measure

$$
\Lambda(\mathrm{d} x, \mathrm{~d} r, \mathrm{~d} w)=\lambda \mathrm{d} x F(\mathrm{~d} r) G_{r}(\mathrm{~d} w),
$$

where $\lambda>0, F$ is a probability measure on $\mathbb{R}_{+}$such that $\int r^{d} F(\mathrm{~d} r)<\infty$, and $r \mapsto G_{r}$ is a probability kernel on $\mathbb{R}_{+}$(a family of probability measures $G_{r}$ on $\mathbb{R}_{+}$indexed by $r$ such that $r \mapsto G_{r}(A)$ is measurable for measurable $\left.A \subset \mathbb{R}_{+}\right)$. A triplet $(x, r, w) \in \Phi$ is identified as a grain with center $x$, radius $r$, and weight $w$. As in Section 2, the constant $\lambda$ is the mean density of grain centers and the probability measure $F$ is the distribution of grain radii. The probability measure $G_{r}$ is the weight distribution of a grain with radius $r$.

We say that two distinct grains are neighbors if they intersect each other, and we denote the set of neighbors of a reference grain $(x, r, w)$ by

$$
N_{x, r, w}=\left\{\left(x^{\prime}, r^{\prime}, w^{\prime}\right) \in \mathbb{R}^{d} \times \mathbb{R}_{+} \times \mathbb{R}_{+} \backslash\{(x, r, w)\}: B_{r^{\prime}}\left(x^{\prime}\right) \cap B_{r}(x) \neq \varnothing\right\} .
$$

The thinning of a weighted Boolean model is defined by $\Phi^{\text {th }}=T(\Phi)$, where

$$
T(\Phi)=\left\{(x, r, w) \in \Phi: w>w^{\prime} \text { for all }\left(x^{\prime}, r^{\prime}, w^{\prime}\right) \in \Phi \cap N_{x, r, w}\right\} .
$$

To rephrase the definition, we say that a grain $\left(x^{\prime}, r^{\prime}, w^{\prime}\right)$ obstructs grain $(x, r, w)$ if $\left(x^{\prime}, r^{\prime}, w^{\prime}\right)$ is a neighbor of $(x, r, w)$ and $w^{\prime} \geq w$. Then, by definition, the thinned germ-grain configuration $\Phi^{\text {th }}$ consists of grains in $\Phi$ which are not obstructed by any other grain in $\Phi$. Note that two overlapping grains with equal weights obstruct each other, and will both be removed. 
The following choices of $G_{r}$ yield the four thinnings which will be analyzed in detail in Sections 5-8.

- Large retained. The weight of each grain is set equal to its radius, so that $G_{r}(\mathrm{~d} w)=$ $\delta_{r}(\mathrm{~d} w)$.

- Random retained. The grains are assigned independent uniformly distributed random weights, so that $G_{r}(\mathrm{~d} w)=\mathbf{1}_{(0,1)}(w) \mathrm{d} w$.

- Small retained. The weight of each grain is set equal to the inverse of its radius, so that $G_{r}(\mathrm{~d} w)=\delta_{1 / r}(\mathrm{~d} w)$.

- Isolated retained. All grains are assigned weight one, so that $G_{r}(\mathrm{~d} w)=\delta_{1}(\mathrm{~d} w)$.

\subsection{Retention probability}

The retention probability of a reference grain $(x, r, w)$ is defined as the probability that $(x, r, w)$ belongs to the germ-grain configuration obtained by thinning the union $\Phi \cup\{(x, r, w)\}$. Because this probability does not depend on $x$ (see Proposition 3.1), we will denote it by

$$
h(r, w)=\mathrm{P}((x, r, w) \in T(\Phi \cup\{(x, r, w)\})) .
$$

The quantity $h(r, w)$ may be regarded as the probability that a typical grain with radius $r$ and weight $w$ in the proposed Boolean model is retained (see, e.g. [20] and [23]). Analogously, the weight-averaged retention probability

$$
h(r)=\int_{\mathbb{R}_{+}} h(r, w) G_{r}(\mathrm{~d} w)
$$

may be regarded as the probability that a typical grain of radius $r$ in the proposed Boolean model is retained. The following result [14, Theorem 2.2] gives a formula for the retention probability. For the reader's convenience, we will include the proof here.

Proposition 3.1. The retention probability of an arbitrary reference grain $(x, r, w) \in \mathbb{R}^{d} \times$ $\mathbb{R}_{+} \times \mathbb{R}_{+}$does not depend on $x$, and is given by

$$
h(r, w)=\exp \left(-\lambda \int_{\mathbb{R}_{+}}\left|B_{r+s}(o)\right| G_{s}[w, \infty) F(\mathrm{~d} s)\right) .
$$

Proof. Fix a reference grain $(x, r, w)$ and define $\Phi^{\prime}=\Phi \cup\{(x, r, w)\}$. By definition, the reference grain belongs to the thinned configuration $T\left(\Phi^{\prime}\right)$ if and only if $w>w^{\prime}$ for all $\left(x^{\prime}, r^{\prime}, w^{\prime}\right) \in N_{x, r, w} \cap \Phi^{\prime}$, where $N_{x, r, w}$ is the neighbor set of $(x, r, w)$ defined by (3.1). Observe that $N_{x, r, w} \cap \Phi^{\prime}=N_{x, r, w} \cap \Phi$, because no grain is its own neighbor by definition. As a consequence, the retention probability can be expressed using the intensity measure of the Poisson process $\Phi$ according to

$$
\mathrm{P}\left((x, r, w) \in T\left(\Phi^{\prime}\right)\right)=\mathrm{P}\left(\Phi\left(A_{x, r, w}\right)=0\right)=\mathrm{e}^{-\Lambda\left(A_{x, r, w}\right)},
$$

where

$$
A_{x, r, w}=\left\{\left(x^{\prime}, r^{\prime}, w^{\prime}\right) \in N_{x, r, w}: w^{\prime} \geq w\right\}
$$

is the set of grains obstructing $(x, r, w)$. The claim now follows because

$$
\begin{aligned}
\Lambda\left(A_{x, r, w}\right) & =\int_{\mathbb{R}^{d}} \int_{\mathbb{R}_{+}} \int_{\mathbb{R}_{+}} \mathbf{1}\left(\left|x-x^{\prime}\right| \leq r+r^{\prime}\right) \mathbf{1}\left(w^{\prime} \geq w\right) G_{r^{\prime}}\left(\mathrm{d} w^{\prime}\right) F\left(\mathrm{~d} r^{\prime}\right) \lambda \mathrm{d} x^{\prime} \\
& =\lambda \int_{\mathbb{R}_{+}}\left|B_{r+r^{\prime}}(o)\right| G_{r^{\prime}}[w, \infty) F\left(\mathrm{~d} r^{\prime}\right) .
\end{aligned}
$$




\subsection{First-order characteristics of the thinned model}

Let us summarize some key formulae about the first-order characteristics of the thinned germ-grain model which were obtained in [1] and [14]. The mean density of grain centers in the thinned model is given by

$$
\lambda_{\text {th }}=\lambda \int_{\mathbb{R}_{+}} h(r) F(\mathrm{~d} r),
$$

where $h(r)$ is the weight-averaged retention probability defined in (3.3), and the radius distribution of a typical grain in the thinned model equals

$$
F_{\text {th }}(r)=1-\frac{\lambda}{\lambda_{\text {th }}} \int_{r}^{\infty} h(s) F(\mathrm{~d} s)
$$

Moreover, the volume fraction of the thinned grain union

$$
X^{\text {th }}=\bigcup_{(x, r, w) \in \Phi^{\text {th }}} B_{r}(x)
$$

is given by

$$
p_{\mathrm{th}}=\lambda \int_{\mathbb{R}_{+}}\left|B_{r}\right| h(r) F(\mathrm{~d} r) .
$$

Note that the quantity $\int h(r) F(\mathrm{~d} r)$ in (3.5) may be regarded as the probability that a randomly chosen grain in the proposed Boolean model is retained by the thinning mechanism.

\subsection{Pair retention probability}

The pair retention probability of a given pair of reference grains $\left(x_{1}, r_{1}, w_{1}\right)$ and $\left(x_{2}, r_{2}, w_{2}\right)$ is defined as the probability that both reference grains belong to the germ-grain configuration obtained by thinning the union $\Phi^{\prime}=\Phi \cup\left\{\left(x_{1}, r_{1}, w_{1}\right),\left(x_{2}, r_{2}, w_{2}\right)\right\}$. Because this probability depends on $x_{1}$ and $x_{2}$ only through their distance (see Proposition 3.2), we will denote it by

$$
h_{2}\left(u, r_{1}, w_{1}, r_{2}, w_{2}\right)=\mathrm{P}\left(\left\{\left(x_{1}, r_{1}, w_{1}\right),\left(x_{2}, r_{2}, w_{2}\right)\right\} \in T\left(\Phi^{\prime}\right)\right),
$$

where $u=\left|x_{1}-x_{2}\right|$. The weight-averaged pair retention probability is defined by

$$
h_{2}\left(u, r_{1}, r_{2}\right)=\int_{\mathbb{R}_{+}} \int_{\mathbb{R}_{+}} h_{2}\left(u, r_{1}, w_{1}, r_{2}, w_{2}\right) G_{r_{1}}\left(\mathrm{~d} w_{1}\right) G_{r_{2}}\left(\mathrm{~d} w_{2}\right) .
$$

Proposition 3.2. The pair retention probability of two reference grains $\left(x_{1}, r_{1}, w_{1}\right)$ and $\left(x_{2}, r_{2}\right.$, $w_{2}$ ) depends on $x_{1}$ and $x_{2}$ only through the distance $u=\left|x_{1}-x_{2}\right|$. For $u \leq r_{1}+r_{2}$, this probability equals 0 , and, for $u>r_{1}+r_{2}$,

$$
h_{2}\left(u, r_{1}, w_{1}, r_{2}, w_{2}\right)=h\left(r_{1}, w_{1}\right) h\left(r_{2}, w_{2}\right) \mathrm{e}^{\tau\left(u, r_{1}, w_{1}, r_{2}, w_{2}\right)},
$$

where $h\left(r_{1}, w_{1}\right)$ and $h\left(r_{2}, w_{2}\right)$ are the retention probabilities defined by (3.4), and

$$
\tau\left(u, r_{1}, w_{1}, r_{2}, w_{2}\right)=\lambda \int_{\mathbb{R}_{+}}\left|B_{r+r_{1}}\left(x_{1}\right) \cap B_{r+r_{2}}\left(x_{2}\right)\right| G_{r}\left[w_{1} \vee w_{2}, \infty\right) F(\mathrm{~d} r)
$$

is the mean number of grains in $\Phi$ which simultaneously obstruct both reference grains. 
Proof. Fix two reference grains $\left(x_{1}, r_{1}, w_{1}\right)$ and $\left(x_{2}, r_{2}, w_{2}\right)$, and assume that they do not overlap, so that $\left|x_{1}-x_{2}\right|>r_{1}+r_{2}$. Denote the pair retention probability, i.e. the right-hand side of (3.8), of these fixed grains by $h_{2}^{*}$ and $\Phi^{\prime}=\Phi \cup\left\{\left(x_{1}, r_{1}, w_{1}\right),\left(x_{2}, r_{2}, w_{2}\right)\right\}$. Recall that grain $\left(x_{1}, r_{1}, w_{1}\right)$ belongs to $T\left(\Phi^{\prime}\right)$ if and only if $w_{1}>w$ for all $(x, r, w) \in N\left(x_{1}, r_{1}, w_{1}\right) \cap \Phi^{\prime}$. Because no grain is its own neighbor by definition, and because the two reference grains are not neighbors, we see that $N\left(x_{1}, r_{1}, w_{1}\right) \cap \Phi^{\prime}=N\left(x_{1}, r_{1}, w_{1}\right) \cap \Phi$. By symmetry, a similar conclusion also holds for the other reference grain.

We conclude that, for $i=1,2$, grain $\left(x_{i}, r_{i}, w_{i}\right)$ is retained if and only if $\Phi\left(A_{i}\right)=0$, where

$$
A_{i}=\left\{(x, r, w) \in N\left(x_{i}, r_{i}, w_{i}\right): w \geq w_{i}\right\}
$$

is the set of grains obstructing $\left(x_{i}, r_{i}, w_{i}\right)$. Now the pair retention probability can be written as

$$
h_{2}^{*}=\mathrm{P}\left(\Phi\left(A_{1} \cup A_{2}\right)=0\right) .
$$

The number of grains in $\Phi \cap\left(A_{1} \cup A_{2}\right)$ is Poisson distributed with mean

$$
\Lambda\left(A_{1} \cup A_{2}\right)=\Lambda\left(A_{1}\right)+\Lambda\left(A_{2}\right)-\Lambda\left(A_{1} \cap A_{2}\right) .
$$

Because $\mathrm{e}^{-\Lambda\left(A_{i}\right)}$ equals the retention probability $h\left(r_{i}, w_{i}\right)$ of grain $\left(x_{i}, r_{i}, w_{i}\right)$ (see Proposition 3.1), we see that

$$
h_{2}^{*}=h\left(r_{1}, w_{1}\right) h\left(r_{2}, w_{2}\right) \mathrm{e}^{\Lambda\left(A_{1} \cap A_{2}\right)} .
$$

The claim now follows after noting that

$$
\Lambda\left(A_{1} \cap A_{2}\right)=\lambda \int_{\mathbb{R}_{+}}\left|B_{r_{1}+r}\left(x_{1}\right) \cap B_{r_{2}+r}\left(x_{2}\right)\right| G_{r}\left[w_{1} \vee w_{2}, \infty\right) F(\mathrm{~d} r) .
$$

A key quantity for analyzing the covariance function of the thinned grain union in Section 4 is the following function, which we will call the retention covariance function. It is defined by

$$
q\left(u, r_{1}, r_{2}\right)=h_{2}\left(u, r_{1}, r_{2}\right)-h\left(r_{1}\right) h\left(r_{2}\right),
$$

where $h(r)$ denotes the weight-averaged retention probability defined in (3.3), and $h_{2}\left(u, r_{1}, r_{2}\right)$ is the weight-averaged pair retention probability defined in (3.9).

Lemma 3.1. The retention covariance function satisfies

$$
\left|q\left(u, r_{1}, r_{2}\right)\right| \leq h\left(r_{1}\right) \wedge h\left(r_{2}\right)
$$

for all $u, r_{1}, r_{2} \geq 0$.

Proof. Fix a pair of reference grains $\left(x_{1}, r_{1}, w_{1}\right)$ and $\left(x_{2}, r_{2}, w_{2}\right)$ having their centers at a distance $u=\left|x_{1}-x_{2}\right|$ apart. Define a weight-dependent version of $q$ by

$$
q\left(u, r_{1}, w_{1}, r_{2}, w_{2}\right)=h_{2}\left(u, r_{1}, w_{1}, r_{2}, w_{2}\right)-h\left(r_{1}, w_{1}\right) h\left(r_{2}, w_{2}\right) .
$$

We will first show that

$$
\left|q\left(u, r_{1}, w_{1}, r_{2}, w_{2}\right)\right| \leq h\left(r_{1}, w_{1}\right),
$$

by separately considering the following two cases.

(i) If $u \leq r_{1}+r_{2}$ then $h_{2}\left(u, r_{1}, w_{1}, r_{2}, w_{2}\right)$ is 0 because the reference grains overlap, and (3.12) follows immediately. 
(ii) If $u>r_{1}+r_{2}$ then by borrowing the notation from the proof of Proposition 3.2 we have, by (3.10),

$$
0 \leq h_{2}\left(u, r_{1}, w_{1}, r_{2}, w_{2}\right)=\mathrm{P}\left(\Phi\left(A_{1} \cup A_{2}\right)=0\right) \leq \mathrm{P}\left(\Phi\left(A_{1}\right)=0\right)=h\left(r_{1}, w_{1}\right) .
$$

As a consequence,

$$
-h\left(r_{1}, w_{1}\right) h\left(r_{2}, w_{2}\right) \leq q\left(u, r_{1}, w_{1}, r_{2}, w_{2}\right) \leq h\left(r_{1}, w_{1}\right)\left(1-h\left(r_{2}, w_{2}\right)\right),
$$

from which (3.12) again follows.

After integrating both sides of (3.12) over the weights, we see that $\left|q\left(u, r_{1}, r_{2}\right)\right| \leq h\left(r_{1}\right)$. By symmetry, the same inequality holds with $r_{1}$ replaced by $r_{2}$, which proves the claim.

\subsection{Covariance function of the thinned grain union}

Let us now consider the covariance function

$$
k_{\text {th }}(z)=\mathrm{P}\left(o \in X^{\text {th }}, \quad z \in X^{\text {th }}\right)-\mathrm{P}\left(o \in X^{\text {th }}\right) \mathrm{P}\left(z \in X^{\text {th }}\right)
$$

of the thinned grain union $X^{\text {th }}$.

Proposition 3.3. The covariance function of the thinned grain union is given by

$$
\begin{aligned}
k_{\mathrm{th}}(z)= & \lambda \int_{\mathbb{R}_{+}}\left|B_{r}(o) \cap B_{r}(z)\right| h(r) F(\mathrm{~d} r) \\
& +\lambda^{2} \int_{\mathbb{R}_{+}} \int_{\mathbb{R}_{+}} \int_{\mathbb{R}^{d}}\left|B_{r_{1}}(o) \cap B_{r_{2}}(x)\right| q\left(|x-z|, r_{1}, r_{2}\right) \mathrm{d} x F\left(\mathrm{~d} r_{1}\right) F\left(\mathrm{~d} r_{2}\right),
\end{aligned}
$$

where $h$ is the weight-averaged retention probability defined in (3.3) and $q$ is the retention covariance function defined in (3.11).

Proof. By using the hard-core property of the thinned configuration we can express the first term on the right-hand side of (3.13) as

$$
\mathrm{P}\left(o \in X^{\text {th }}, z \in X^{\text {th }}\right)=S_{1}(z)+S_{2}(z),
$$

where $S_{1}(z)$ is the probability that a single grain in $\Phi^{\text {th }}$ simultaneously covers $o$ and $z$, and $S_{2}(z)$ is the probability that $o$ and $z$ are covered by distinct grains in $\Phi^{\text {th }}$.

To write down an analytical expression for $S_{1}(z)$, recall first that, by the hard-core property, the indicator function of $X^{\text {th }}$ can be written as

$$
\mathbf{1}_{X^{\mathrm{th}}}(y)=\sum_{(x, r, w) \in \Phi} f_{y}(\Phi ; x, r, w),
$$

where $f_{y}(\Phi ; x, r, w)=\mathbf{1}\left(y \in B_{r}(x)\right) \mathbf{1}_{T(\Phi)}(x, r, w)$ is the indicator for the event that a grain $(x, r, w)$ covers $y$ and is contained in $\Phi^{\text {th }}$. Then

$$
S_{1}(z)=\mathrm{E} \sum_{(x, r, w) \in \Phi} f_{o}(\Phi ; x, r, w) f_{z}(\Phi ; x, r, w) .
$$

Using Mecke's formula [20, Theorem 3.2.5] and the definition of the retention probability given in (3.2), we obtain

$$
S_{1}(z)=\int \mathbf{1}_{B_{r}(o)}(x) \mathbf{1}_{B_{r}(z)}(x) h(r, w) \mathrm{d} \Lambda
$$


where $\mathrm{d} \Lambda=\lambda \mathrm{d} x F(\mathrm{~d} r) G_{r}(\mathrm{~d} w)$ is the intensity measure of the Poisson process $\Phi$. Now using the weight-averaged retention probability defined in (3.3) and integrating over $x$, we see that

$$
S_{1}(z)=\lambda \int_{\mathbb{R}_{+}}\left|B_{r}(o) \cap B_{r}(z)\right| h(r) F(\mathrm{~d} r),
$$

which corresponds to the first integral on the right-hand side of (3.14).

The probability that $o$ and $z$ are covered by distinct grains in $\Phi^{\text {th }}$ can analogously be written as

$$
S_{2}(z)=\mathrm{E} \sum_{\substack{\left(x_{1}, r_{1}, w_{1}\right) \in \Phi \\\left(x_{2}, r_{2}, w_{2}\right) \in \Phi \\\left(x_{1}, r_{1}, w_{1}\right) \neq\left(x_{2}, r_{2}, w_{2}\right)}} f_{o}\left(\Phi ; x_{1}, r_{1}, w_{1}\right) f_{z}\left(\Phi ; x_{2}, r_{2}, w_{2}\right) .
$$

Using the Slivnyak-Mecke formula [20, Corollary 3.2.3] and the definition of the pair retention probability given in (3.8), the probability $S_{2}(z)$ can be written as

$$
S_{2}(z)=\int \mathbf{1}_{B_{r_{1}}(o)}\left(x_{1}\right) \mathbf{1}_{B_{r_{2}}(z)}\left(x_{2}\right) h_{2}\left(\left|x_{1}-x_{2}\right|, r_{1}, w_{1}, r_{2}, w_{2}\right) \mathrm{d} \Lambda_{1} \mathrm{~d} \Lambda_{2},
$$

where $\mathrm{d} \Lambda_{i}$ is short for $\lambda \mathrm{d} x_{i} F\left(\mathrm{~d} r_{i}\right) G_{r_{i}}\left(\mathrm{~d} w_{i}\right)$, the intensity measure of $\Phi$. By making the change of variables $x_{2}=x_{1}+z-x$ so that $\mathbf{1}_{B_{r_{2}}(z)}\left(x_{2}\right)$ becomes $\mathbf{1}_{B_{r_{2}}(x)}\left(x_{1}\right)$ and integrating over $x_{1}$, we see that

$$
S_{2}(z)=\lambda^{2} \iiint\left|B_{r_{1}}(o) \cap B_{r_{2}}(x)\right| h_{2}\left(|x-z|, r_{1}, r_{2}\right) \mathrm{d} x F\left(\mathrm{~d} r_{1}\right) F\left(\mathrm{~d} r_{2}\right),
$$

where we also took the weight averaging inside the pair retention probability $h_{2}$ as in (3.9).

The validity of the claim now follows after representing $\mathrm{P}\left(z \in X^{\text {th }}\right)=p_{\text {th }}$ using (3.7) and the identity $\left|B_{r_{1}}(o)\right|\left|B_{r_{2}}(o)\right|=\int\left|B_{r_{1}}(o) \cap B_{r_{2}}(x)\right| \mathrm{d} x$ to note that

$$
p_{\mathrm{th}}^{2}=\lambda^{2} \iiint\left|B_{r_{1}}(o) \cap B_{r_{2}}(x)\right| h\left(r_{1}\right) h\left(r_{2}\right) \mathrm{d} x F\left(\mathrm{~d} r_{1}\right) F\left(\mathrm{~d} r_{2}\right),
$$

and finally combining $S_{2}(z)$ and $p_{\text {th }}^{2}$ with the help of the retention covariance function (3.11).

\subsection{Two-point correlation function of thinned grain centers}

The two-point correlation function $\xi_{\text {th }}(z)$ of the random point configuration $\Phi_{g}^{\text {th }}=$ $\left\{x:(x, r, w) \in \Phi^{\text {th }}\right\}$ of the thinned grain centers is defined as a function which satisfies

$$
\operatorname{cov}\left(\Phi_{g}^{\mathrm{th}}(A), \Phi_{g}^{\mathrm{th}}(B)\right)=\lambda_{\text {th }}^{2} \int_{A} \int_{B} \xi_{\mathrm{th}}(x-y) \mathrm{d} x \mathrm{~d} y
$$

for all disjoint and bounded measurable sets $A, B \subset \mathbb{R}^{d}$, assuming that such a function exists. This function, which in our case depends only on $|z|$, describes how much more $\left(\xi_{\text {th }}(z)>0\right)$ or less $\left(\xi_{\text {th }}(z)<0\right)$ likely it is to observe a point at a distance $|z|$ from a typical point, compared to observing a point in an arbitrary location. The two-point correlation function is related to the pair-correlation function $g_{\text {th }}$ commonly used in statistics (see, e.g. [23]), via the formula $\xi_{\text {th }}(z)=g_{\text {th }}(z)-1$.

Proposition 3.4. The two-point correlation function of the thinned grain centers is given by

$$
\xi_{\text {th }}(z)=\frac{\lambda^{2}}{\lambda_{\text {th }}^{2}} \int_{\mathbb{R}_{+}} \int_{\mathbb{R}_{+}} q\left(|z|, r_{1}, r_{2}\right) F\left(\mathrm{~d} r_{1}\right) F\left(\mathrm{~d} r_{2}\right),
$$

where $\lambda_{\text {th }}$ is the thinned germ density defined in (3.5), and $q$ is the retention covariance function defined in (3.11). 
Proof. First note that $\Phi_{g}^{\text {th }}(A)=\Phi^{\text {th }}\left(A \times \mathbb{R}_{+} \times \mathbb{R}_{+}\right)=\sum_{(x, r, w) \in \Phi} \mathbf{1}((x, r, w) \in T(\Phi))$. Using this, we can write, for disjoint $A, B \subset \mathbb{R}^{d}$,

$$
\begin{aligned}
& \mathrm{E} \Phi_{g}^{\mathrm{th}}(A) \Phi_{g}^{\mathrm{th}}(B) \\
& =\mathrm{E} \sum_{\substack{\left(x_{1}, r_{1}, w_{1}\right) \in \Phi \\
\left(x_{2}, r_{2}, w_{2}\right) \in \Phi}} \mathbf{1}_{A}\left(x_{1}\right) \mathbf{1}_{B}\left(x_{2}\right) \mathbf{1}\left(\left(x_{1}, r_{1}, w_{1}\right) \in T(\Phi)\right) \mathbf{1}\left(\left(x_{2}, r_{2}, w_{2}\right) \in T(\Phi)\right) .
\end{aligned}
$$

Because $A$ and $B$ are disjoint, we may now apply the Slivnyak-Mecke formula [20, Corollary 3.2.3] to obtain

$$
\mathrm{E} \Phi_{g}^{\mathrm{th}}(A) \Phi_{g}^{\mathrm{th}}(B)=\int \mathbf{1}_{A}\left(x_{1}\right) \mathbf{1}_{B}\left(x_{2}\right) h_{2}\left(\left|x_{1}-x_{2}\right|, r_{1}, w_{1}, r_{2}, w_{2}\right) \mathrm{d} \Lambda_{1} \mathrm{~d} \Lambda_{2},
$$

where $\mathrm{d} \Lambda_{i}$ is short for $\lambda \mathrm{d} x_{i} F\left(\mathrm{~d} r_{i}\right) G_{r_{i}}\left(\mathrm{~d} w_{i}\right)$, the intensity measure of the Poisson process $\Phi$, and $h_{2}$ is the pair retention probability defined in (3.8). Using the weight-averaged pair retention probability defined in (3.9), we may write

$$
\mathrm{E} \Phi_{g}^{\mathrm{th}}(A) \Phi_{g}^{\mathrm{th}}(B)=\lambda^{2} \int_{A} \int_{B} \int_{\mathbb{R}_{+}} \int_{\mathbb{R}_{+}} h_{2}\left(|x-y|, r_{1}, r_{2}\right) F\left(\mathrm{~d} r_{1}\right) F\left(\mathrm{~d} r_{2}\right) \mathrm{d} x \mathrm{~d} y .
$$

In a similar fashion we obtain

$$
\mathrm{E} \Phi_{g}^{\mathrm{th}}(A)=\lambda \int_{A} \int_{\mathbb{R}_{+}} h(r) F(\mathrm{~d} r) \mathrm{d} x
$$

where $h(r)$ is the weight-averaged retention probability defined in (3.3).

Combining the two expressions above and recalling the definition of the retention covariance function (3.11) yields

$$
\operatorname{cov}\left(\Phi_{g}^{\mathrm{th}}(A), \Phi_{g}^{\mathrm{th}}(B)\right)=\lambda^{2} \int_{A} \int_{B} \int_{\mathbb{R}_{+}} \int_{\mathbb{R}_{+}} q\left(|x-y|, r_{1}, r_{2}\right) F\left(\mathrm{~d} r_{1}\right) F\left(\mathrm{~d} r_{2}\right) \mathrm{d} x \mathrm{~d} y .
$$

We can now define

$$
\xi_{\text {th }}(z)=\frac{\lambda^{2}}{\lambda_{\text {th }}^{2}} \int_{\mathbb{R}_{+}} \int_{\mathbb{R}_{+}} q\left(|z|, r_{1}, r_{2}\right) F\left(\mathrm{~d} r_{1}\right) F\left(\mathrm{~d} r_{2}\right),
$$

which satisfies (3.15)

\section{Long-range behavior of second-order characteristics}

In this section we assume that the grain radius distribution $F$ of the proposed Boolean model follows a power law with tail exponent $\alpha>d$, by which we mean that the complementary cumulative distribution function $\bar{F}(r)=1-F(r)$ is regularly varying at $\infty$ with exponent $-\alpha$. In this case we can write

$$
\bar{F}(r)=\ell(r) r^{-\alpha},
$$

where the function $\ell$ is slowly varying at $\infty$ (see Appendix A for details). 


\subsection{Asymptotic covariance}

The following result describes the covariance function of the grain union for thinnings where large grains have a small retention probability. This result will be used to prove Theorems 5.1 and 6.1 in Sections 5 and 6.

Proposition 4.1. Assume that the radius distribution $F$ follows a power law with tail exponent $\alpha>d$. Assume that the weight-averaged retention probability $h(r)$ decays to 0 as $r \rightarrow \infty$, and that there exists a function $q_{\infty}: \mathbb{R}_{+} \times \mathbb{R}_{+} \rightarrow \mathbb{R}$ such that

$$
c=\lambda^{2}\left|B_{1}\right|^{2} \int_{\mathbb{R}_{+}} \int_{\mathbb{R}_{+}} r_{1}^{d} r_{2}^{d} q_{\infty}\left(r_{1}, r_{2}\right) F\left(\mathrm{~d} r_{1}\right) F\left(\mathrm{~d} r_{2}\right)
$$

is finite and nonzero, and that, for any $r_{1}, r_{2} \geq 0$, the retention covariance function defined in (3.11) decays according to

$$
q\left(|z|, r_{1}, r_{2}\right) \sim q_{\infty}\left(r_{1}, r_{2}\right) \bar{F}(|z|)|z|^{d} \quad \text { as }|z| \rightarrow \infty .
$$

Then the covariance function of the thinned grain union decays according to

$$
k_{\mathrm{th}}(z) \sim c \bar{F}(|z|)|z|^{d} \quad \text { as }|z| \rightarrow \infty .
$$

To prove Proposition 4.1, we need detailed results about the retention probabilities. The following lemma allows us to use dominated convergence on a part of the domain.

Lemma 4.1. Assume that the radius distribution $F$ follows a power law with tail exponent $\alpha>d$. Then there exist constants $c>0$ and $m>0$ such that

$$
0 \leq q\left(|x-z|, r_{1}, r_{2}\right) \leq c|z|^{d} \bar{F}(|z|)
$$

for all $x, z \in \mathbb{R}^{d}$ and all $r_{1}, r_{2} \geq 0$ such that $|x|<r_{1}+r_{2},|x-z| \geq 2\left(r_{1}+r_{2}\right)$, and $|z|>m$.

Proof. Let $c_{1}$ and $u_{1}$ be the constants from Lemma B.2. Using the assumption that the function $\bar{F}$ follows a power law with tail exponent $\alpha$, choose $u_{2}$ such that $\bar{F}(2 / 3 r) / \bar{F}(r) \leq$ $2\left(\frac{2}{3}\right)^{-\alpha}$ for all $r>u_{1}$. Choose $u_{3}$ such that $\lambda c_{1} r^{d} \bar{F}(r) \leq 1$ for all $r>u_{2}$. Note that $|x| \leq$ $r_{1}+r_{2} \leq \frac{1}{2}|x-z|$ implies that $|z| \leq|x|+|x-z| \leq \frac{3}{2}|x-z|$, and let $m=\max \left\{u_{1}, \frac{3}{2} u_{2}, \frac{3}{2} u_{3}\right\}$. Using Proposition 3.2 and the definition of $m$, we have, for all $|z|>m$,

$$
\begin{aligned}
q\left(|x-z|, r_{1}, r_{2}\right) & \leq \exp \left(\lambda \int_{\mathbb{R}_{+}}\left|B_{r_{1}+r}(o) \cap B_{r_{2}+r}(|x-z|)\right| F(\mathrm{~d} r)\right)-1 \\
& \leq \exp \left(\lambda c_{1}|x-z|^{d} \bar{F}(|x-z|)\right)-1 \\
& \leq 2 \lambda c_{1}|x-z|^{d} \bar{F}(|x-z|) .
\end{aligned}
$$

Note that $|x-z| \leq|x|+|z| \leq \frac{1}{2}|x-z|+|z|$ implies that $|x-z| \leq 2|z|$, and that $\bar{F}$ is a decreasing function. Now, for $c=4\left(\frac{2}{3}\right)^{-\alpha} 2^{d} \lambda c_{1}$ and $|z|>m$, we have

$$
q\left(|x-z|, r_{1}, r_{2}\right) \leq 2 \lambda c_{1}(2|z|)^{d} \bar{F}\left(\frac{2}{3}|z|\right) \leq c|z|^{d} \bar{F}(|z|) .
$$

Lemma 4.2. Fix $z \in \mathbb{R}^{d}$, and define

$$
A(z)=\left\{\left(x, r_{1}, r_{2}\right) \in \mathbb{R}^{d} \times \mathbb{R}_{+} \times \mathbb{R}_{+}:|x-z| \leq 2\left(r_{1}+r_{2}\right)\right\} .
$$


Then the retention covariance function $q$ satisfies

$$
\begin{gathered}
\iiint_{A(z)}\left|B_{r_{1}}(o) \cap B_{r_{2}}(x)\right|\left|q\left(|x-z|, r_{1}, r_{2}\right)\right| \mathrm{d} x F\left(\mathrm{~d} r_{1}\right) F\left(\mathrm{~d} r_{2}\right) \\
\quad \leq 2\left|B_{1}\right|^{2}\left(\int_{\mathbb{R}_{+}} r^{d} F(\mathrm{~d} r)\right)\left(\int_{|z| / 6}^{\infty} r^{d} F(\mathrm{~d} r)\right) \sup _{r>|z| / 6} h(r),
\end{gathered}
$$

where $h(r)$ is the weight-averaged retention probability defined in (3.3).

Proof. Define $\mathrm{d} \mu$ as shorthand for $\mathrm{d} x F\left(\mathrm{~d} r_{1}\right) F\left(\mathrm{~d} r_{2}\right)$, and denote the integrand by $f_{z}\left(x, r_{1}, r_{2}\right)$. Observe that $f_{z}$ vanishes outside the set $A_{0}=\left\{\left(x, r_{1}, r_{2}\right):|x|<r_{1}+r_{2}\right\}$. Observe also that $A(z) \cap A_{0} \subset A_{1}(z) \cup A_{2}(z)$, where $A_{i}(z)=\left\{\left(x, r_{1}, r_{2}\right): r_{i}>|z| / 6\right\}$. As a consequence,

$$
\int_{A(z)} f_{z} \mathrm{~d} \mu=\int_{A(z) \cap A_{0}} f_{z} \mathrm{~d} \mu \leq \int_{A_{1}(z)} f_{z} \mathrm{~d} \mu+\int_{A_{2}(z)} f_{z} \mathrm{~d} \mu=2 \int_{A_{1}(z)} f_{z} \mathrm{~d} \mu,
$$

where the last equality is due to the symmetry of $f_{z}$ with respect to its last two arguments. Recall that $\left|q\left(|x-z|, r_{1}, r_{2}\right)\right| \leq h\left(r_{1}\right)$ by Lemma 3.1. This inequality implies that

$$
\begin{aligned}
\int_{A_{1}(z)} f_{z} \mathrm{~d} \mu & \leq \iiint \mathbf{1}_{(|z| / 6, \infty)}\left(r_{1}\right)\left|B_{r_{1}}(o) \cap B_{r_{2}}(x)\right| h\left(r_{1}\right) \mathrm{d} x F\left(\mathrm{~d} r_{1}\right) F\left(\mathrm{~d} r_{2}\right) \\
& \leq J(z) \sup _{r>|z| / 6} h(r),
\end{aligned}
$$

where

$$
\begin{aligned}
J(z) & =\iiint \mathbf{1}_{(|z| / 6, \infty)}\left(r_{1}\right)\left|B_{r_{1}}(o) \cap B_{r_{2}}(x)\right| \mathrm{d} x F\left(\mathrm{~d} r_{1}\right) F\left(\mathrm{~d} r_{2}\right) \\
& =\left|B_{1}\right|^{2}\left(\int_{\mathbb{R}_{+}} r^{d} F(\mathrm{~d} r)\right)\left(\int_{|z| / 6}^{\infty} r^{d} F(\mathrm{~d} r)\right) .
\end{aligned}
$$

Proof of Proposition 4.1. By Proposition 3.3 we can write

$$
\frac{k_{\mathrm{th}}(z)}{\bar{F}(|z|)|z|^{d}}=\lambda I_{1}(z)+\lambda^{2}\left(I_{2}(z)+I_{3}(z)\right),
$$

where

$$
I_{1}(z)=\left(\bar{F}(|z|)|z|^{d}\right)^{-1} \int_{\mathbb{R}_{+}}\left|B_{r}(o) \cap B_{r}(z)\right| h(r) F(\mathrm{~d} r),
$$

and where

$$
\begin{aligned}
& I_{2}(z)=\iiint_{A_{z}} f_{z}\left(x, r_{1}, r_{2}\right) \mathrm{d} x F\left(\mathrm{~d} r_{1}\right) F\left(\mathrm{~d} r_{2}\right), \\
& I_{3}(z)=\iiint_{A_{z}^{c}} f_{z}\left(x, r_{1}, r_{2}\right) \mathrm{d} x F\left(\mathrm{~d} r_{1}\right) F\left(\mathrm{~d} r_{2}\right)
\end{aligned}
$$

denote the integrals of the function

$$
f_{z}\left(x, r_{1}, r_{2}\right)=\left|B_{r_{1}}(o) \cap B_{r_{2}}(x)\right|\left(\frac{q\left(|x-z|, r_{1}, r_{2}\right)}{\bar{F}(|z|)|z|^{d}}\right)
$$

over the set

$$
A_{z}=\left\{\left(x, r_{1}, r_{2}\right):|x-z| \leq 2\left(r_{1}+r_{2}\right)\right\}
$$

and its complement, respectively. 
The integral $I_{1}(z) \rightarrow 0$ as $|z| \rightarrow \infty$ by Lemma B.3, because $h(r) \rightarrow 0$ as $r \rightarrow \infty$ by assumption.

We will next show that $I_{2}(z) \rightarrow 0$ as well. We apply Lemma 4.2 to conclude that

$$
\left|I_{2}(z)\right| \leq c_{2}\left(|z|^{d} \bar{F}(|z|)\right)^{-1}\left(\int_{|z| / 6}^{\infty} r^{d} F(\mathrm{~d} r)\right) \sup _{r>|z| / 6} h(r),
$$

where $c_{2}=2\left|B_{1}\right|^{2} \int r^{d} F(\mathrm{~d} r)$. The right-hand side above tends to 0 as $|z| \rightarrow \infty$, because $h(r) \rightarrow 0$ as $r \rightarrow \infty$, and because the integral on the right-hand side above is asymptotically equivalent to a constant multiple of $|z|^{d} \bar{F}(|z|)$ by Lemma A.1.

To analyze the limiting behavior of $I_{3}(z)$ as $|z| \rightarrow \infty$, note that assumption (4.1) and Lemma A. 2 imply that, for any $x, r_{1}, r_{2}$,

$$
q\left(|x-z|, r_{1}, r_{2}\right) \sim q_{\infty}\left(r_{1}, r_{2}\right)|x-z|^{d} \bar{F}(|x-z|) \sim q_{\infty}\left(r_{1}, r_{2}\right)|z|^{d} \bar{F}(|z|) .
$$

By the definition of $A_{z}$, it thus follows that

$$
f_{z}\left(x, r_{1}, r_{2}\right) \mathbf{1}_{A_{z}^{c}}\left(x, r_{1}, r_{2}\right) \rightarrow q_{\infty}\left(r_{1}, r_{2}\right)\left|B_{r_{1}}(o) \cap B_{r_{2}}(x)\right|
$$

as $|z| \rightarrow \infty$. Moreover, by Lemma 4.1 , there exists a constant $c_{3}$ such that

$$
\left|f_{z}\left(x, r_{1}, r_{2}\right) \mathbf{1}_{A_{z}^{c}}\left(x, r_{1}, r_{2}\right)\right| \leq c_{3}\left|B_{r_{1}}(o) \cap B_{r_{2}}(x)\right|
$$

for all $x, r_{1}, r_{2}$ and all large enough $z$. Because the right-hand side above is integrable with respect to $\mathrm{d} x F\left(\mathrm{~d} r_{1}\right) F\left(\mathrm{~d} r_{2}\right)$, Lebesgue's dominated convergence theorem shows that

$$
\begin{aligned}
\lim _{|z| \rightarrow \infty} I_{3}(z) & =\iiint q_{\infty}\left(r_{1}, r_{2}\right)\left|B_{r_{1}}(o) \cap B_{r_{2}}(x)\right| \mathrm{d} x F\left(\mathrm{~d} r_{1}\right) F\left(\mathrm{~d} r_{2}\right) \\
& =\left|B_{1}\right|^{2} \iint q_{\infty}\left(r_{1}, r_{2}\right) r_{1}^{d} r_{2}^{d} F\left(\mathrm{~d} r_{1}\right) F\left(\mathrm{~d} r_{2}\right),
\end{aligned}
$$

which completes the proof of Proposition 4.1.

\subsection{Asymptotic two-point correlation}

The following result describes the two-point correlation function of the grain centers for general weight-based thinnings. This result will be used to prove Theorems 5.1, 6.1, and 7.1 in Sections 5, 6, and 7.

Proposition 4.2. Assume that the radius distribution $F$ follows a power law with tail exponent $\alpha>d$. Assume also that there exists a function $q_{\infty}: \mathbb{R}_{+} \times \mathbb{R}_{+} \rightarrow \mathbb{R}$ such that

$$
c=\frac{\lambda^{2}}{\lambda_{\text {th }}^{2}} \int_{\mathbb{R}_{+}} \int_{\mathbb{R}_{+}} q_{\infty}\left(r_{1}, r_{2}\right) F\left(\mathrm{~d} r_{1}\right) F\left(\mathrm{~d} r_{2}\right)
$$

is finite and nonzero, and that, for any $r_{1}, r_{2} \geq 0$, the retention covariance function defined in (3.11) decays according to

$$
q\left(|z|, r_{1}, r_{2}\right) \sim q_{\infty}\left(r_{1}, r_{2}\right)|z|^{d} \bar{F}(|z|) \quad \text { as }|z| \rightarrow \infty .
$$

Then the two-point correlation of the thinned grain centers decays according to

$$
\xi_{\text {th }}(z) \sim c|z|^{d} \bar{F}(|z|) \quad \text { as }|z| \rightarrow \infty .
$$


Proof. Using (3.16), we can write

$$
\frac{\xi_{\mathrm{th}}(z)}{|z|^{d} \bar{F}(|z|)}=\frac{\lambda^{2}}{\lambda_{\mathrm{th}}^{2}}\left(I_{1}(z)+I_{2}(z)\right)
$$

where

$$
\begin{aligned}
& I_{1}(z)=\iint_{A_{z}} f_{z}\left(r_{1}, r_{2}\right) F\left(\mathrm{~d} r_{1}\right) F\left(\mathrm{~d} r_{2}\right), \\
& I_{2}(z)=\iint_{A_{z}^{c}} f_{z}\left(r_{1}, r_{2}\right) F\left(\mathrm{~d} r_{1}\right) F\left(\mathrm{~d} r_{2}\right)
\end{aligned}
$$

denote the integrals of

$$
f_{z}\left(r_{1}, r_{2}\right)=\frac{q\left(|z|, r_{1}, r_{2}\right)}{|z|^{d} \bar{F}(|z|)}
$$

over the set

$$
A_{z}=\left\{\left(r_{1}, r_{2}\right) \in \mathbb{R}_{+} \times \mathbb{R}_{+}: r_{1}+r_{2}>\frac{1}{2}|z|\right\}
$$

and its complement, respectively.

Observe that $A_{z} \subset A_{1}(z) \cup A_{2}(z)$, where $A_{i}(z)=\left\{\left(r_{1}, r_{2}\right): r_{i}>|z| / 4\right\}$, and that $|q| \leq 1$ by Lemma 3.1. As a consequence,

$$
\int_{A_{z}}\left|q\left(|z|, r_{1}, r_{2}\right)\right| F\left(\mathrm{~d} r_{1}\right) F\left(\mathrm{~d} r_{2}\right) \leq(F \times F)\left(A_{1}(z)\right)+(F \times F)\left(A_{2}(z)\right)=2 \bar{F}\left(\frac{|z|}{4}\right),
$$

which implies that $I_{1}(z) \rightarrow 0$ as $|z| \rightarrow \infty$.

Note that $f_{z}\left(r_{1}, r_{2}\right) \mathbf{1}_{A_{z}^{c}}\left(r_{1}, r_{2}\right) \rightarrow q_{\infty}\left(r_{1}, r_{2}\right)$ by assumption (4.2) and the definition of $A_{z}$. By Lemma 4.1, $f_{z}\left(r_{1}, r_{2}\right) \mathbf{1}_{A_{z}^{c}}\left(r_{1}, r_{2}\right)$ is bounded for large $z$ uniformly on $r_{1}$ and $r_{2}$. Lebesgue's dominated convergence theorem then shows that

$$
\lim _{|z| \rightarrow \infty} I_{2}(z)=\iint q_{\infty}\left(r_{1}, r_{2}\right) F(\mathrm{~d} r) F(\mathrm{~d} s) .
$$

\section{Isolated grains retained}

In this section we study the thinning where only isolated grains are retained. In the general framework of Section 3, this is achieved by assigning unit weight to every grain, so that $G_{r}(\mathrm{~d} w)=\delta_{1}(\mathrm{~d} w)$. For nonrandom equally sized grains, this corresponds to the classical Matérn type-I thinning.

Theorem 5.1. Assume that the radius distribution $F$ follows a power law with tail exponent $\alpha>d$, so that $1-F(r)=\ell(r) r^{-\alpha}$ for some slowly varying function $\ell$. Then the thinned radius distribution is bounded by

$$
\bar{F}_{\text {th }}(r) \leq \frac{\lambda}{\lambda_{\text {th }}} \mathrm{e}^{-\lambda\left|B_{1}\right| r^{d}},
$$

the covariance function of the thinned grain union decays according to

$$
k_{\mathrm{th}}(z) \sim \lambda c_{\alpha, d} p_{\mathrm{th}}^{2} \ell(|z|)|z|^{-(\alpha-d)} \quad \text { as }|z| \rightarrow \infty,
$$

and the two-point correlation function of the thinned grain centers according to

$$
\xi_{\text {th }}(z) \sim \lambda c_{\alpha, d} \ell(|z|)|z|^{-(\alpha-d)} \quad \text { as }|z| \rightarrow \infty,
$$

where the constant $c_{\alpha, d}$ is given in (B.2). 
Proof. Because the weights are deterministic, the retention probabilities have simple formulae:

$$
h(r)=h(r, 1)=\exp \left(-\lambda\left|B_{1}\right| \int_{\mathbb{R}_{+}}(r+s)^{d} F(\mathrm{~d} s)\right)
$$

and

$$
h_{2}\left(|z|, r_{1}, r_{2}\right)=h\left(r_{1}\right) h\left(r_{2}\right) \exp \left(\lambda \int_{\mathbb{R}_{+}}\left|B_{r_{1}+s}(o) \cap B_{r_{2}+s}(z)\right| F(\mathrm{~d} s)\right) .
$$

The tail of the thinned radius distribution (3.6) is

$$
\bar{F}_{\text {th }}(r)=\frac{\lambda}{\lambda_{\text {th }}} \int_{r}^{\infty} h(s) F(\mathrm{~d} s) \leq \frac{\lambda}{\lambda_{\text {th }}} h(r) \leq \frac{\lambda}{\lambda_{\text {th }}} \mathrm{e}^{-\lambda\left|B_{1}\right| r^{d}} .
$$

To show the claim for the covariance and two-point correlation functions, we will use Proposition 4.1 and Proposition 4.2, respectively. For that, we need to show that (4.1) holds. By Lemma B.1 we have, for the average intersection volume in $h_{2}$ above,

$$
\int_{\mathbb{R}_{+}}\left|B_{r_{1}+s}(o) \cap B_{r_{2}+s}(z)\right| F(\mathrm{~d} s) \sim c|z|^{d} \bar{F}(|z|) .
$$

Because the right-hand side goes to 0 as $|z| \rightarrow \infty$, we can use the fact that $\lim _{t \rightarrow \infty}\left(\mathrm{e}^{t}-1\right) / t=1$ to obtain (4.1) with

$$
q_{\infty}\left(r_{1}, r_{2}\right)=h\left(r_{1}\right) h\left(r_{2}\right) \lambda c_{\alpha, d} .
$$

Using Proposition 4.1, we find that $k_{\text {th }}(z) \sim c_{1}|z|^{d} \bar{F}(|z|)$. Using the formula for volume fraction (3.7), we also find the constant $c_{1}=\lambda p_{\text {th }}^{2} c_{\alpha, d}$. Similarly, by Proposition 4.2 we find that $\xi_{\text {th }}(z) \sim c_{2}|z|^{d} \bar{F}(|z|)$. With the help of germ density (3.5) we have $c_{2}=\lambda c_{\alpha, d}$.

\section{Random grains retained}

Here we assume that each grain in the proposed Boolean model is assigned a random weight independently of the other grains, according to some continuous distribution function. Continuity ensures that there will be no tie breaks. Because the shape of the weight distribution does not affect the retention probabilities considered here, as long as it is continuous, we may without loss of generality assume that $G_{r}(\mathrm{~d} w)=\mathbf{1}_{(0,1)}(w) \mathrm{d} w$, the uniform distribution on $(0,1)$. Note that, for nonrandom equally sized grains, this corresponds to the classical Matérn type-II thinning.

Theorem 6.1. Assume that the radius distribution $F$ follows a power law with tail exponent $\alpha>d$, so that $1-F(r)=\ell(r) r^{-\alpha}$ for some slowly varying function $\ell$. Then the thinned radius distribution decays according to

$$
\bar{F}_{\text {th }}(r) \sim\left(\lambda_{\text {th }}\left|B_{1}\right|\right)^{-1} \frac{\alpha}{\alpha+d} \ell(r) r^{-(\alpha+d)} \quad \text { as } r \rightarrow \infty,
$$

the covariance function of the thinned grain union according to

$$
k_{\mathrm{th}}(z) \sim c_{1} \ell(|z|)|z|^{-(\alpha-d)} \text { as }|z| \rightarrow \infty,
$$

and the two-point correlation function of the thinned grain centers according to

$$
\xi_{\text {th }}(z) \sim c_{2} \ell(|z|)|z|^{-(\alpha-d)} \quad \text { as }|z| \rightarrow \infty,
$$

for some $c_{1}, c_{2} \in(0, \infty)$. 
Proof. The retention probability of a grain with radius $r$ and weight $w \in(0,1)$ is

$$
h(r, w)=\exp \left(-\lambda \int_{\mathbb{R}_{+}} G_{R}[w, \infty)\left|B_{r+s}(o)\right| F(\mathrm{~d} s)\right)=\mathrm{e}^{-\lambda(1-w) b(r)},
$$

where

$$
b(r)=\int_{\mathbb{R}_{+}}\left|B_{r+s}(o)\right| F(\mathrm{~d} s) .
$$

The weight-averaged retention probability thus equals

$$
h(r)=\int_{0}^{1} \mathrm{e}^{-\lambda(1-w) b(r)} \mathrm{d} w=\frac{1-\mathrm{e}^{-\lambda b(r)}}{\lambda b(r)} .
$$

Because $b(r) \rightarrow \infty$ as $r \rightarrow \infty$, it follows that $\lim _{r \rightarrow \infty} h(r)=0$. The first condition of Proposition 4.1 is thus satisfied.

Note that $b(r) /\left|B_{r}\right|=\int_{\mathbb{R}_{+}}(1+s / r)^{d} F(\mathrm{~d} s)$ and so, by dominated convergence, $b(r) \sim\left|B_{r}\right|$ as $r \rightarrow \infty$, which implies that

$$
h(r) \sim(\lambda b(r))^{-1} \sim\left(\lambda\left|B_{1}\right| r^{d}\right)^{-1} .
$$

By Lemma A.4, the thinned radius distribution (3.6) is

$$
\bar{F}_{\text {th }}(r)=\frac{\lambda}{\lambda_{\text {th }}} \int_{r}^{\infty} h(s) F(\mathrm{~d} s) \sim\left(\lambda_{\text {th }}\left|B_{1}\right|\right)^{-1} \int_{r}^{\infty} s^{-d} F(\mathrm{~d} s) .
$$

Furthermore, by Lemma A.1,

$$
\bar{F}_{\text {th }}(r) \sim\left(\lambda_{\text {th }}\left|B_{1}\right|\right)^{-1} \frac{\alpha}{\alpha+d} r^{-d} \bar{F}(r) .
$$

The pair retention probability equals

$$
\begin{aligned}
& h_{2}\left(|z|, r_{1}, r_{2}, w_{1}, w_{2}\right) \\
& \quad=h\left(r_{1}, w_{1}\right) h\left(r_{2}, w_{2}\right) \exp \left(\lambda \int_{\mathbb{R}_{+}} G_{R}\left[w_{1} \vee w_{2}, \infty\right)\left|B_{r_{1}+s}(o) \cap B_{r_{2}+s}(z)\right| F(\mathrm{~d} s)\right) \\
& =\exp \left(-\lambda\left(1-w_{1}\right) b\left(r_{1}\right)-\lambda\left(1-w_{2}\right) b\left(r_{2}\right)+\lambda\left(1-w_{1} \vee w_{2}\right) a_{z}\left(r_{1}, r_{2}\right)\right),
\end{aligned}
$$

where

$$
a_{z}\left(r_{1}, r_{2}\right)=\int_{\mathbb{R}_{+}}\left|B_{r_{1}+s}(o) \cap B_{r_{2}+s}(z)\right| F(\mathrm{~d} s) .
$$

From this expression we see that the retention covariance function defined in (3.11) equals

$$
q\left(|z|, r_{1}, r_{2}\right)=\int_{0}^{1} \int_{0}^{1} \mathrm{e}^{-\lambda b\left(r_{1}\right)\left(1-w_{1}\right)} \mathrm{e}^{-\lambda b\left(r_{2}\right)\left(1-w_{2}\right)}\left(\mathrm{e}^{\lambda\left(1-w_{1} \vee w_{2}\right) a_{z}\left(r_{1}, r_{2}\right)}-1\right) \mathrm{d} w_{1} \mathrm{~d} w_{2} .
$$

As $|z| \rightarrow \infty$, Lemma B.1 shows that the term in parentheses above is asymptotically equivalent to

$$
\mathrm{e}^{\lambda\left(1-w_{1} \vee w_{2}\right) a_{z}\left(r_{1}, r_{2}\right)}-1 \sim \lambda\left(1-w_{1} \vee w_{2}\right) c_{\alpha, d} \bar{F}(|z|)|z|^{d} .
$$

With the help of the bound $\left|\mathrm{e}^{t}-1\right| \leq(\mathrm{e}-1) t$ for $t \in[0,1]$, we may use dominated convergence to conclude that

$$
q\left(|z|, r_{1}, r_{2}\right) \sim q_{\infty}\left(r_{1}, r_{2}\right)|z|^{d} \bar{F}(|z|) \quad \text { as }|z| \rightarrow \infty
$$


where

$$
q_{\infty}\left(r_{1}, r_{2}\right)=\lambda c_{\alpha, d} \int_{0}^{1} \int_{0}^{1}\left(1-w_{1} \vee w_{2}\right) \mathrm{e}^{-\lambda b\left(r_{1}\right)\left(1-w_{1}\right)} \mathrm{e}^{-\lambda b\left(r_{2}\right)\left(1-w_{2}\right)} \mathrm{d} w_{1} \mathrm{~d} w_{2} .
$$

Now, by Proposition 4.1, it follows that

$$
k_{\mathrm{th}}(z) \sim c_{1} \bar{F}(|z|)|z|^{d} \quad \text { as }|z| \rightarrow \infty,
$$

where

$$
c_{1}=\lambda^{2}\left|B_{1}\right|^{2} \int_{\mathbb{R}_{+}} \int_{\mathbb{R}_{+}} r_{1}^{d} r_{2}^{d} q_{\infty}\left(r_{1}, r_{2}\right) F\left(\mathrm{~d} r_{1}\right) F\left(\mathrm{~d} r_{2}\right) .
$$

The constant $c_{1}$ is finite because $q_{\infty}\left(r_{1}, r_{2}\right) \leq \lambda c_{\alpha, d}$ for all $r_{1}, r_{2}$. The fact that $c_{1}$ is strictly positive is easily seen by inspecting the expression of $q_{\infty}\left(r_{1}, r_{2}\right)$.

Similarly, Proposition 4.2 shows that

$$
\xi_{\text {th }}(z) \sim c_{2}|z|^{d} \bar{F}(|z|),
$$

where

$$
c_{2}=\frac{\lambda^{2}}{\lambda_{\text {th }}^{2}} \int_{\mathbb{R}_{+}} \int_{\mathbb{R}_{+}} q_{\infty}\left(r_{1}, r_{2}\right) F\left(\mathrm{~d} r_{1}\right) F\left(\mathrm{~d} r_{2}\right) .
$$

A similar reasoning as for $c_{1}$ shows that the constant $c_{2}$ is finite and strictly positive.

\section{Large grains retained}

A thinning which favors large grains is obtained by letting the weight of each grain be equal to its radius, so that $G_{r}(\mathrm{~d} w)=\delta_{r}(\mathrm{~d} w)$.

Theorem 7.1. Assume that the radius distribution $F$ follows a power law with tail exponent $\alpha>d$, so that $1-F(r)=\ell(r) r^{-\alpha}$ for some slowly varying function $\ell$. Then the thinned radius distribution decays according to

$$
\bar{F}_{\mathrm{th}}(r) \sim \frac{\lambda}{\lambda_{\mathrm{th}}} \ell(r) r^{-\alpha} \text { as } r \rightarrow \infty,
$$

the covariance function of the thinned grain union according to

$$
k_{\mathrm{th}}(z) \sim \lambda c_{\alpha, d}\left(1-p_{\mathrm{th}}\right)^{2} \ell(|z|)|z|^{-(\alpha-d)} \text { as }|z| \rightarrow \infty,
$$

and the two-point correlation function of the thinned grain centers according to

$$
\xi_{\text {th }}(z) \sim \lambda c_{\alpha, d} \ell(|z|)|z|^{-(\alpha-d)} \quad \text { as }|z| \rightarrow \infty,
$$

where the constant $c_{\alpha, d}$ is given in (B.2).

Proof. Because the weight of each grain is equal to its radius, the weight-averaged retention probability $h(r)$ is equal to $h(r, w)$ with $w$ taking on the value $r$. By Proposition 3.1, the retention probability is given by

$$
h(r)=\exp \left(-\lambda \int_{\mathbb{R}_{+}}\left|B_{r+s}\right| \mathbf{1}_{[r, \infty)}(s) F(\mathrm{~d} s)\right) .
$$


Because the integrand above tends to 0 as $r \rightarrow \infty$, and the integrand is bounded by the $F(\mathrm{~d} s)$-integrable function $\left|B_{2 s}\right|$, dominated convergence implies that $\lim _{r \rightarrow \infty} h(r)=1$. By Lemma A.4, the tail of the thinned radius distribution (3.6) satisfies

$$
\bar{F}_{\text {th }}(r)=\frac{\lambda}{\lambda_{\text {th }}} \int_{r}^{\infty} h(s) F(\mathrm{~d} s) \sim \frac{\lambda}{\lambda_{\text {th }}} \int_{r}^{\infty} F(\mathrm{~d} s)=\frac{\lambda}{\lambda_{\text {th }}} \bar{F}(r) .
$$

To analyze the long-range behaviors of $k_{\mathrm{th}}(z)$ and $\xi_{\mathrm{th}}(z)$, let us first investigate the longrange behavior of the retention covariance function $q\left(|z|, r_{1}, r_{2}\right)$ defined in (3.11). Using Proposition 3.2, we find that

$$
q\left(|z|, r_{1}, r_{2}\right)=h\left(r_{1}\right) h\left(r_{2}\right)\left(\mathbf{1}\left(r_{1}+r_{2}<|z|\right) \mathrm{e}^{\tau\left(|z|, r_{1}, r_{2}\right)}-1\right),
$$

where

$$
\tau\left(|z|, r_{1}, r_{2}\right)=\lambda \int_{\mathbb{R}_{+}} \mathbf{1}_{\left[r_{1} \vee r_{2}, \infty\right)}(s)\left|B_{r_{1}+s}(o) \cap B_{r_{2}+s}(z)\right| F(\mathrm{~d} s) .
$$

When $|z|>3\left(r_{1}+r_{2}\right)$, we may replace the region of integration above with the full positive real line, so that with the help of Lemma B.1 we find that

$$
\tau\left(|z|, r_{1}, r_{2}\right)=\lambda \int_{\mathbb{R}_{+}}\left|B_{r_{1}+s}(o) \cap B_{r_{2}+s}(z)\right| F(\mathrm{~d} s) \sim \lambda c_{\alpha, d}|z|^{d} \bar{F}(|z|)
$$

as $|z| \rightarrow \infty$. Because $\mathrm{e}^{t}-1 \sim t$ for small $t$, we conclude using (7.1) that

$$
q\left(|z|, r_{1}, r_{2}\right) \sim q_{\infty}\left(r_{1}, r_{2}\right)|z|^{d} \bar{F}(|z|)
$$

where

$$
q_{\infty}\left(r_{1}, r_{2}\right)=\lambda c_{\alpha, d} h\left(r_{1}\right) h\left(r_{2}\right) .
$$

The claim for the two-point correlation function $\xi_{\text {th }}(z)$ now follows by using Proposition 4.2, after noting that the constant in Proposition 4.2 is

$$
\frac{\lambda^{2}}{\lambda_{\mathrm{th}}^{2}} \iint q_{\infty}\left(r_{1}, r_{2}\right) F\left(\mathrm{~d} r_{1}\right) F\left(\mathrm{~d} r_{2}\right)=\lambda \lambda_{\mathrm{th}}^{-2} c_{\alpha, d}\left(\lambda \int h(r) F(\mathrm{~d} r)\right)^{2}=\lambda c_{\alpha, d} .
$$

We will now move on to the part concerning the covariance function $k_{\mathrm{th}}(z)$ of the thinned grain union. Note that, because $h(r)$ does not vanish as $r \rightarrow \infty$, we cannot use Proposition 4.1 to deduce the long-range behavior of $k_{\mathrm{th}}(z)$. Instead, we will proceed by directly analyzing the integral building blocks of $k_{\mathrm{th}}(z)$ in high precision. Let us start by rewriting (3.14) as

$$
k_{\mathrm{th}}(z)=\lambda I_{0}(z)+\lambda^{2}\left(I_{1}(z)+I_{2}(z)+I_{3}(z)\right),
$$

where

$$
\begin{gathered}
I_{0}(z)=\int_{\mathbb{R}_{+}}\left|B_{r}(o) \cap B_{r}(z)\right| h(r) F(\mathrm{~d} r), \\
I_{j}(z)=\iiint_{A_{j}^{z}}\left|B_{r_{1}}(o) \cap B_{r_{2}}(x)\right| q\left(|x-z|, r_{1}, r_{2}\right) \mathrm{d} x F\left(\mathrm{~d} r_{1}\right) F\left(\mathrm{~d} r_{2}\right), \quad j=1,2,3,
\end{gathered}
$$


and

$$
\begin{aligned}
& A_{1}^{z}=\left\{\left(x, r_{1}, r_{2}\right):|x|<r_{1}+r_{2}, r_{1}+r_{2}<\frac{1}{2}|x-z|\right\}, \\
& A_{2}^{z}=\left\{\left(x, r_{1}, r_{2}\right):|x|<r_{1}+r_{2}, \frac{1}{2}|x-z|<r_{1}+r_{2}<|x-z|\right\}, \\
& A_{3}^{z}=\left\{\left(x, r_{1}, r_{2}\right):|x|<r_{1}+r_{2},|x-z|<r_{1}+r_{2}\right\} .
\end{aligned}
$$

The first term $I_{0}(z) \lesssim c_{\alpha, d}|z|^{d} \bar{F}(|z|)$ by Lemma B.1. Note that the integrand in $I_{0}(z)$ vanishes for $r \leq|z| / 2$ so that

$$
I_{0}(z) \geq \inf _{r \geq|z| / 2} h(r) \int_{\mathbb{R}_{+}}\left|B_{r}(o) \cap B_{r}(z)\right| F(\mathrm{~d} r) .
$$

Using Lemma B.1 and the fact that $h(r) \rightarrow 1$ as $r \rightarrow \infty$, we conclude that

$$
I_{0}(z) \sim c_{\alpha, d}|z|^{d} \bar{F}(|z|) .
$$

Next, we will prove that

$$
I_{1}(z) \sim \lambda^{-1} c_{\alpha, d} p_{\mathrm{th}}^{2}|z|^{d} \bar{F}(|z|) .
$$

By Lemma 4.1, the function

$$
\left(x, r_{1}, r_{2}\right) \mapsto \frac{q\left(|x-z|, r_{1}, r_{2}\right)}{|z|^{d} \bar{F}(|z|)} \mathbf{1}_{A_{1}^{z}}\left(x, r_{1}, r_{2}\right)
$$

is positive and bounded by a constant which does not depend on $z$. Because $\left|B_{r_{1}}(o) \cap B_{r_{2}}(x)\right|$ is integrable with respect to $\mathrm{d} x F\left(\mathrm{~d} r_{1}\right) F\left(\mathrm{~d} r_{2}\right)$, Lebesgue's dominated convergence theorem shows that

$$
\lim _{z \rightarrow \infty} \frac{I_{1}(z)}{|z|^{d} \bar{F}(|z|)}=\iiint\left|B_{r_{1}}(o) \cap B_{r_{2}}(x)\right|\left(\lim _{z \rightarrow \infty} \frac{q\left(|x-z|, r_{1}, r_{2}\right)}{|z|^{d} \bar{F}(|z|)} \mathbf{1}_{A_{1}^{z}}\right) \mathrm{d} x F\left(\mathrm{~d} r_{1}\right) F\left(\mathrm{~d} r_{2}\right) .
$$

Using (7.3) and the definition of $A_{1}^{z}$, the limit on the right-hand side equals $q_{\infty}\left(r_{1}, r_{2}\right)$. Plugging in the expression for $q_{\infty}\left(r_{1}, r_{2}\right)$ given in (7.4) and recalling the formula for the volume fraction of the thinned grain union $p_{\text {th }}$ given in (3.7), we find that

$$
\begin{aligned}
\lim _{|z| \rightarrow \infty} \frac{I_{1}(z)}{|z|^{d} \bar{F}(|z|)} & =\lambda c_{\alpha, d} \iiint\left|B_{r_{1}}(o) \cap B_{r_{2}}(x)\right| h\left(r_{1}\right) h\left(r_{2}\right) \mathrm{d} x F\left(\mathrm{~d} r_{1}\right) F\left(\mathrm{~d} r_{2}\right) \\
& =\lambda c_{\alpha, d} \iint\left|B_{1}\right|^{2} r_{1}^{d} r_{2}^{d} h\left(r_{1}\right) h\left(r_{2}\right) F\left(\mathrm{~d} r_{1}\right) F\left(\mathrm{~d} r_{2}\right) \\
& =\lambda^{-1} c_{\alpha, d} p_{\mathrm{th}}^{2},
\end{aligned}
$$

which proves the validity of (7.6).

Now we will prove that

$$
\frac{I_{2}(z)}{|z|^{d} \bar{F}(|z|)} \rightarrow 0 \quad \text { as }|z| \rightarrow \infty
$$

First, using the bound $\left|B_{r_{1}+s}(o) \cap B_{r_{2}+s}(x-z)\right| \leq\left|B_{r_{1}+s}\right| \leq\left|B_{2 s}\right|$ for $s \geq r_{1} \vee r_{2}$, we find that the function $\tau$ defined in (7.2) is bounded by

$$
\tau\left(|x-z|, r_{1}, r_{2}\right) \leq \lambda\left|B_{1}\right| 2^{d} \int \mathbf{1}_{\left[r_{1} \vee r_{2}, \infty\right)}(s) s^{d} F(\mathrm{~d} s) .
$$


Observe next that

$$
|z| \leq 3\left(r_{1}+r_{2}\right) \leq 6\left(r_{1} \vee r_{2}\right)
$$

for all $\left(x, r_{1}, r_{2}\right) \in A_{2}^{z}$, so that $r_{1} \vee r_{2}$ is large when $|z|$ is large. As a consequence, we see, by Lemma A.1 and Lemma A.3, that, for all $\left(x, r_{1}, r_{2}\right) \in A_{2}^{z}$ and all large enough $z$,

$$
\tau\left(|x-z|, r_{1}, r_{2}\right) \leq 2 \lambda\left|B_{1}\right| 2^{d}\left(r_{1} \vee r_{2}\right)^{d} \bar{F}\left(r_{1} \vee r_{2}\right) \leq 4 \lambda\left|B_{1}\right| 2^{d}\left(\frac{1}{6}|z|\right)^{d} \bar{F}\left(\frac{1}{6}|z|\right) .
$$

Because $\mathrm{e}^{t}-1 \leq(\mathrm{e}-1) t$ for $t \in[0,1]$, formula (7.1) combined with the above inequality shows that, for all $\left(x, r_{1}, r_{2}\right) \in A_{2}^{z}$ and all large enough $z$,

$$
0 \leq q\left(|x-z|, r_{1}, r_{2}\right) \leq c_{1}|z|^{d} \bar{F}\left(\frac{1}{6}|z|\right),
$$

where $c_{1}=4(\mathrm{e}-1) \lambda\left|B_{1}\right| 3^{-d}$. Therefore,

$$
0 \leq I_{2}(z) \leq c_{1}|z|^{d} \bar{F}\left(\frac{|z|}{6}\right) \iiint_{A_{2}^{z}}\left|B_{r_{1}}(o) \cap B_{r_{2}}(x)\right| \mathrm{d} x F\left(\mathrm{~d} r_{1}\right) F\left(\mathrm{~d} r_{2}\right) .
$$

Note that $A_{2}^{z} \subset A_{21}^{z} \cup A_{22}^{z}$, where $A_{2 i}^{z}=\left\{\left(x, r_{1}, r_{2}\right): r_{i} \geq|z| / 6\right\}, i=1,2$. By symmetry of the integrand with respect to $r_{1}$ and $r_{2}$,

$$
\begin{aligned}
0 \leq I_{2}(z) & \leq 2 c_{1}|z|^{d} \bar{F}\left(\frac{|z|}{6}\right) \int_{\mathbb{R}_{+}} \int_{|z| / 6}^{\infty} \int_{\mathbb{R}^{d}}\left|B_{r_{1}}(o) \cap B_{r_{2}}(x)\right| \mathrm{d} x F\left(\mathrm{~d} r_{1}\right) F\left(\mathrm{~d} r_{2}\right) \\
& =2 c_{1}|z|^{d} \bar{F}\left(\frac{|z|}{6}\right)\left|B_{1}\right|^{2}\left(\int r^{d} F(\mathrm{~d} r)\right)\left(\int_{|z| / 6}^{\infty} r^{d} F(\mathrm{~d} r)\right),
\end{aligned}
$$

which shows the validity of (7.7).

It remains to be shown that

$$
I_{3}(z) \sim-2 \frac{p_{\text {th }}}{\lambda} c_{\alpha, d}|z|^{d} \bar{F}(|z|) .
$$

To do that, we first fix a small $\varepsilon \in\left(0, \frac{1}{4}\right)$. Note that, by (7.1), the retention covariance function equals $q\left(|x-z|, r_{1}, r_{2}\right)=-h\left(r_{1}\right) h\left(r_{2}\right)$ for $\left(x, r_{1}, r_{2}\right) \in A_{3}^{z}$. Note also that, for fixed $r_{1}$ and $r_{2}$, the $x$-slice of $A_{3}^{z}$ is

$$
\left\{x:\left(x, r_{1}, r_{2}\right) \in A_{3}^{z}\right\}=B_{r_{1}+r_{2}}(z) \cap B_{r_{1}+r_{2}}(o) .
$$

Because $\left|B_{r_{1}}(o) \cap B_{r_{2}}(x)\right|$ vanishes for $x$ outside $B_{r_{1}+r_{2}}(o)$, we may represent $I_{3}(z)$ according to

$$
\begin{aligned}
I_{3}(z) & =-\iiint_{A_{3}^{z}}\left|B_{r_{1}}(o) \cap B_{r_{2}}(x)\right| h\left(r_{1}\right) h\left(r_{2}\right) \mathrm{d} x F\left(\mathrm{~d} r_{1}\right) F\left(\mathrm{~d} r_{2}\right) \\
& =-\iint_{C_{3}^{z}} \int_{B_{r_{1}+r_{2}}(z)}\left|B_{r_{1}}(o) \cap B_{r_{2}}(x)\right| \mathrm{d} x h\left(r_{1}\right) h\left(r_{2}\right) F\left(\mathrm{~d} r_{1}\right) F\left(\mathrm{~d} r_{2}\right),
\end{aligned}
$$

where

$$
C_{3}^{z}=\left\{\left(r_{1}, r_{2}\right):|z| \leq 2\left(r_{1}+r_{2}\right)\right\} .
$$

Next we split $I_{3}(z)$ into three parts:

$$
I_{3}(z)=-\left(I_{31}(z)+I_{32}(z)+I_{33}(z)\right) .
$$


Here

$$
I_{3 j}(z)=\iint_{C_{3 j}^{z}} \int_{B_{r_{1}+r_{2}}(z)}\left|B_{r_{1}}(o) \cap B_{r_{2}}(x)\right| \mathrm{d} x h\left(r_{1}\right) h\left(r_{2}\right) F\left(\mathrm{~d} r_{1}\right) F\left(\mathrm{~d} r_{2}\right)
$$

for $j=1,2,3$ and

$$
\begin{aligned}
C_{31}^{z} & =C_{311}^{z} \cup C_{312}^{z}, \\
C_{311}^{z} & =\left\{\left(r_{1}, r_{2}\right): 0 \leq r_{1} \leq \varepsilon|z|, \frac{1}{2}|z| \leq r_{2}\right\}, \\
C_{312}^{z} & =\left\{\left(r_{1}, r_{2}\right): 0 \leq r_{2} \leq \varepsilon|z|, \frac{1}{2}|z| \leq r_{1}\right\}, \\
C_{32}^{z} & =C_{331}^{z} \cup C_{332}^{z}, \\
C_{321}^{z} & =\left\{\left(r_{1}, r_{2}\right): 0 \leq r_{1} \leq \varepsilon|z|, \frac{1}{2}|z|-r_{1} \leq r_{2} \leq \frac{1}{2}|z|\right\}, \\
C_{322}^{z} & =\left\{\left(r_{1}, r_{2}\right): 0 \leq r_{2} \leq \varepsilon|z|, \frac{1}{2}|z|-r_{2} \leq r_{1} \leq \frac{1}{2}|z|\right\}, \\
C_{33}^{z} & =[\varepsilon|z|, \infty)^{2} \cap C_{3}^{z} .
\end{aligned}
$$

A change of variables shows that

$$
\int_{B_{r_{1}+r_{2}}(z)}\left|B_{r_{1}}(o) \cap B_{r_{2}}(x)\right| \mathrm{d} x=\int_{B_{r_{1}}(o)}\left|B_{r_{2}}(z) \cap B_{r_{1}+r_{2}}(x)\right| \mathrm{d} x,
$$

so that we can express the integral $I_{31}(z)$ more conveniently as

$$
I_{31}(z)=\iint_{C_{31}^{z}} \int_{B_{r_{1}}(o)}\left|B_{r_{2}}(z) \cap B_{r_{1}+r_{2}}(x)\right| \mathrm{d} x h\left(r_{1}\right) h\left(r_{2}\right) F\left(\mathrm{~d} r_{1}\right) F\left(\mathrm{~d} r_{2}\right) .
$$

By symmetry we can write $I_{31}(z)=2 I_{311}(z)$, where $I_{311}(z)$ is a modification of $I_{31}(z)$ with the region of integration $C_{31}^{z}$ replaced by $C_{311}^{z}$. To analyze the long-range behavior of $I_{311}(z)$, let us split it according to $I_{311}(z)=J_{1}(z)+J_{2}(z)$, where

$$
J_{1}(z)=\int_{0}^{\varepsilon|z|} \int_{|z| / 2}^{\infty} \int_{B_{r_{1}}(o)}\left|B_{r_{2}}(z) \cap B_{r_{2}}(o)\right| \mathrm{d} x h\left(r_{2}\right) F\left(\mathrm{~d} r_{2}\right) h\left(r_{1}\right) F\left(\mathrm{~d} r_{1}\right),
$$

and where $J_{2}(z)=I_{311}(z)-J_{1}(z)$. Because the integrand of $J_{1}(z)$ does not depend on $x$, we can rewrite the integral as

$$
J_{1}(z)=\left(\int_{0}^{\varepsilon|z|}\left|B_{r}\right| h(r) F(\mathrm{~d} r)\right)\left(\int_{|z| / 2}^{\infty}\left|B_{r}(z) \cap B_{r}(o)\right| h(r) F(\mathrm{~d} r)\right) .
$$

The first integral on the right-hand side satisfies

$$
\int_{0}^{|z| \varepsilon}\left|B_{1}\right| r^{d} h(r) F(\mathrm{~d} r) \sim \int_{\mathbb{R}_{+}}\left|B_{1}\right| r^{d} h(r) F(\mathrm{~d} r)=\lambda^{-1} p_{\text {th }},
$$

where $p_{\text {th }}$ is the volume fraction of the thinned grain union given by (3.7). Note that, because the intersection in the second integral vanishes for $r<|z| / 2$, we can apply (7.5) to conclude that

$$
J_{1}(z) \sim \lambda^{-1} p_{\text {th }} c_{\alpha, d}|z|^{d} \bar{F}(|z|) .
$$

The rest of the proof constitutes showing that the remaining three parts of $I_{3}(z)$ are negligible. We start by showing that $J_{2} \geq 0$ and

$$
\limsup _{|z| \rightarrow \infty} \frac{J_{2}(z)}{|z|^{d} \bar{F}(|z|)} \leq\left|B_{1}\right|^{2}\left((1+2 \varepsilon)^{d}-1\right)\left(\int r^{d} F(\mathrm{~d} r)\right) \frac{\alpha}{\alpha-d} 2^{\alpha-d} .
$$


First we need a bound for the difference of the intersections in $J_{2}(z)$. Fix $x \in B_{r_{1}}(o)$ and $\left(r_{1}, r_{2}\right) \in C_{311}^{z}$. Because $|x| \leq r_{1}$, we have $B_{r_{2}}(o) \subset B_{r_{1}+r_{2}}(x)$, which implies that the integrand in $J_{2}(z)$ is bounded by

$$
\begin{aligned}
0 & \leq\left|B_{r_{2}}(z) \cap B_{r_{1}+r_{2}}(x)\right|-\left|B_{r_{2}}(z) \cap B_{r_{2}}(o)\right| \\
& =\left|B_{r_{2}}(z) \cap\left(B_{r_{1}+r_{2}}(x) \backslash B_{r_{2}}(o)\right)\right| \\
& \leq\left|B_{r_{1}+r_{2}}(x)\right|-\left|B_{r_{2}}(o)\right| \\
& =\left|B_{1}\right|\left(\left(1+\frac{r_{1}}{r_{2}}\right)^{d}-1\right) r_{2}^{d} \\
& \leq\left|B_{1}\right|\left((1+2 \varepsilon)^{d}-1\right) r_{2}^{d},
\end{aligned}
$$

where the last inequality is due to $r_{1} \leq \varepsilon|z|$ and $|z| / 2 \leq r_{2}$. This bound and $h(r) \leq 1$ now imply that

$$
\begin{aligned}
0 & \leq J_{2}(z) \\
& \leq \int_{0}^{\varepsilon|z|} \int_{|z| / 2}^{\infty} \int_{B_{r_{1}}(o)}\left|B_{1}\right|\left((1+2 \varepsilon)^{d}-1\right) r_{2}^{d} \mathrm{~d} x h\left(r_{2}\right) F\left(\mathrm{~d} r_{2}\right) h\left(r_{1}\right) F\left(\mathrm{~d} r_{1}\right) \\
& \leq\left|B_{1}\right|^{2}\left((1+2 \varepsilon)^{d}-1\right)\left(\int_{0}^{\varepsilon|z|} r^{d} F(\mathrm{~d} r)\right)\left(\int_{|z| / 2}^{\infty} r^{d} F(\mathrm{~d} r)\right) .
\end{aligned}
$$

Now using Lemma A.1 proves the claim.

We will now show that $I_{32} \geq 0$ and

$$
\limsup _{|z| \rightarrow \infty} \frac{I_{32}(z)}{|z|^{d} \bar{F}(|z|)} \leq 2\left(\int r^{d} F(\mathrm{~d} r)\right)\left|B_{1}\right|^{2}\left[(1-2 \varepsilon)^{d-\alpha}-1\right] \frac{\alpha}{\alpha-d} 2^{\alpha-d} .
$$

By symmetry, $I_{32}(z)=2 I_{321}(z)$, where

$$
I_{321}(z)=\iint_{C_{321}^{z}} \int_{B_{r_{1}+r_{2}}(z)}\left|B_{r_{1}}(o) \cap B_{r_{2}}(x)\right| \mathrm{d} x h\left(r_{1}\right) h\left(r_{2}\right) F\left(\mathrm{~d} r_{1}\right) F\left(\mathrm{~d} r_{2}\right) .
$$

Note that $C_{321}^{z} \subset[0, \varepsilon|z|] \times[|z| / 2(1-2 \varepsilon),|z| / 2]$. Also, approximating $B_{r_{1}+r_{2}}(z)$ by $\mathbb{R}^{d}$ and recalling that $h(r) \leq 1$ we have

$$
\begin{aligned}
I_{321}(z) & \leq \int_{0}^{|z| \varepsilon} \int_{(|z| / 2)(1-2 \varepsilon)}^{|z| / 2}\left|B_{1}\right|^{2} r_{1}^{d} r_{2}^{d} F\left(\mathrm{~d} r_{2}\right) F\left(\mathrm{~d} r_{1}\right) \\
& =\left|B_{1}\right|^{2}\left(\int_{0}^{|z| \varepsilon} r^{d} F(\mathrm{~d} r)\right)\left(\int_{(|z| / 2)(1-2 \varepsilon)}^{\infty} r^{d} F(\mathrm{~d} r)-\int_{|z| / 2}^{\infty} r^{d} F(\mathrm{~d} r)\right) .
\end{aligned}
$$

Again, using Lemma A.1 implies the claim.

For the last part $I_{33}(z)$, we have first the simple bound

$$
\begin{aligned}
I_{33}(z) & \leq \int_{\varepsilon|z|}^{\infty} \int_{\varepsilon|z|}^{\infty} \int_{B_{r_{1}+r_{2}}(z)}\left|B_{r_{1}}(o) \cap B_{r_{2}}(x)\right| \mathrm{d} x h\left(r_{1}\right) h\left(r_{2}\right) F\left(\mathrm{~d} r_{1}\right) F\left(\mathrm{~d} r_{2}\right) \\
& \leq \int_{\varepsilon|z|}^{\infty} \int_{\varepsilon|z|}^{\infty}\left|B_{1}\right|^{2} r_{1}^{d} r_{2}^{d} F\left(\mathrm{~d} r_{1}\right) F\left(\mathrm{~d} r_{2}\right) \\
& =\left|B_{1}\right|^{2}\left(\int_{\varepsilon|z|}^{\infty} r^{d} F(\mathrm{~d} r)\right)^{2} .
\end{aligned}
$$


Using Lemma A.1 once more, the above bound implies that

$$
\frac{I_{33}(z)}{|z|^{d} \bar{F}(z)} \rightarrow 0 \quad \text { as }|z| \rightarrow \infty .
$$

Adding together all the parts of $I_{3}(z)$ we have

$$
\limsup _{|z| \rightarrow \infty} \frac{I_{3}(z)}{|z|^{d} \bar{F}(|z|)}=-2 \frac{p_{\text {th }}}{\lambda} c_{\alpha, d}
$$

and

$$
\liminf _{|z| \rightarrow \infty} \frac{I_{3}(z)}{|z|^{d} \bar{F}(|z|)} \geq-2 \frac{p_{\text {th }}}{\lambda} c_{\alpha, d}-\delta(\varepsilon)
$$

where

$$
\delta(\varepsilon)=2\left|B_{1}\right|^{2}\left(\int r^{d} F(\mathrm{~d} r)\right) \frac{\alpha}{\alpha-d} 2^{\alpha-d}\left(\left((1+2 \varepsilon)^{d}-1\right)+\left((1-2 \varepsilon)^{d-\alpha}-1\right)\right) .
$$

Letting $\varepsilon \rightarrow 0$ shows the validity of (7.8) and concludes the proof.

\section{Small grains retained}

In this section we study a thinning which favors small grains. This thinning is obtained by setting the weight of each grain to the inverse of its radius, so that $G_{r}(\mathrm{~d} w)=\delta_{1 / r}(\mathrm{~d} w)$. The following theorem shows that the thinned radius distribution and the key second-order characteristics decay rapidly to 0 , regardless of the tail behavior of the original radius distribution $F$. Note that here, unlike in Theorems 5.1-7.1, there is no need to assume anything on the shape of the radius distribution $F$.

Theorem 8.1. Assume that the radius distribution $F$ satisfies $\int r^{d} F(\mathrm{~d} r)<\infty$. Then the thinned radius distribution is bounded by

$$
\bar{F}_{\text {th }}(r) \leq \frac{\lambda}{\lambda_{\text {th }}} \mathrm{e}^{-\lambda\left|B_{1}\right| r^{d} / 2},
$$

the covariance function of the thinned grain union by

$$
\left|k_{\mathrm{th}}(z)\right| \leq \mathrm{e}^{-\lambda\left|B_{1}\right| c|z|^{d}}
$$

and the two-point correlation function of the thinned grain centers by

$$
\left|\xi_{\text {th }}(z)\right| \leq \mathrm{e}^{-\lambda\left|B_{1}\right| c|z|^{d}}
$$

for all large values of $r$ and $z$, where $c \in(0, \infty)$.

Proof. Now the weight-averaged retention probability $h(r)$ is equal to the retention probability of a reference grain with radius $r$ and weight $1 / r$. Using Proposition 3.1, we find that

$$
h(r)=\exp \left(-\lambda \int_{0}^{r}\left|B_{r+s}(o)\right| F(\mathrm{~d} s)\right) .
$$


From this expression we see that $h$ decreases monotonically to 0 as $r$ grows, and that $h(r) \leq$ $\exp \left(-\frac{1}{2} \lambda\left|B_{1}\right| r^{d}\right)$ for all large enough $r$ so that $F(r) \geq \frac{1}{2}$. Proposition 3.2 further shows that the weight-averaged pair retention probability equals

$$
h_{2}\left(|z|, r_{1}, r_{2}\right)=h\left(r_{1}\right) h\left(r_{2}\right) \exp \left(\lambda \int \mathbf{1}_{\left[0, r_{1} \wedge r_{2}\right]}(r)\left|B_{r_{1}+r}(o) \cap B_{r_{2}+r}(z)\right| F(\mathrm{~d} r)\right)
$$

for $|z|>r_{1}+r_{2}$.

To analyze the covariance function of the thinned grain union, recall that

$$
\begin{aligned}
k_{\mathrm{th}}(z)= & \lambda \int\left|B_{r}(o) \cap B_{r}(z)\right| h(r) F(\mathrm{~d} r) \\
& +\lambda^{2} \iiint\left|B_{r_{1}}(o) \cap B_{r_{2}}(x)\right| q\left(|x-z|, r_{1}, r_{2}\right) \mathrm{d} x F\left(\mathrm{~d} r_{1}\right) F\left(\mathrm{~d} r_{2}\right),
\end{aligned}
$$

where $q\left(u, r_{1}, r_{2}\right)=h_{2}\left(u, r_{1}, r_{2}\right)-h\left(r_{1}\right) h\left(r_{2}\right)$. Because $\left|B_{r}(o) \cap B_{r}(z)\right| \leq\left|B_{1}\right| r^{d} \mathbf{1}(r>$ $|z| / 2)$, the first term on the right-hand side of (8.2) is bounded from above by

$$
\lambda\left|B_{1}\right|\left(\int_{|z| / 2}^{\infty} r^{d} F(\mathrm{~d} r)\right) \sup _{r>|z| / 2} h(r) .
$$

Note that $q\left(|x-z|, r_{1}, r_{2}\right)$ vanishes for $|x-z|>2\left(r_{1}+r_{2}\right)$, because the integral in (8.1) vanishes for $|z| \geq 2\left(r_{1}+r_{2}\right)$. This is why the integration of the second term in (8.2) can be restricted to the set $A(z)=\left\{\left(x, r_{1}, r_{2}\right):|x-z| \leq 2\left(r_{1}+r_{2}\right)\right\}$. Now, using Lemma 4.2, the absolute value of the second term in (8.2) is bounded from above by

$$
2 \lambda^{2}\left|B_{1}\right|^{2}\left(\int_{\mathbb{R}_{+}} r^{d} F(\mathrm{~d} r)\right)\left(\int_{|z| / 6}^{\infty} r^{d} F(\mathrm{~d} r)\right) \sup _{r>|z| / 6} h(r) .
$$

As consequence, we find that

$$
\left|k_{\mathrm{th}}(z)\right| \leq\left(\lambda m_{1}+2 \lambda^{2} m_{1}^{2}\right) \sup _{r>|z| / 6} h(r),
$$

where $m_{1}=\left|B_{1}\right| \int r^{d} F(\mathrm{~d} r)$ is the mean volume of a grain. Therefore,

$$
\left|k_{\mathrm{th}}(z)\right| \leq\left(\lambda m_{1}+2 \lambda^{2} m_{1}^{2}\right) \mathrm{e}^{-\lambda\left|B_{1}\right|(|z| / 6)^{d} / 2}
$$

for all large enough $z$ such that $F(|z|) \geq \frac{1}{2}$. A similar analysis can be carried out for the two-point correlation function.

A typical radius has tail probabilities (3.6)

$$
\bar{F}_{\text {th }}(r)=\frac{\lambda}{\lambda_{\text {th }}} \int_{r}^{\infty} h(s) F(\mathrm{~d} s) \leq \frac{\lambda}{\lambda_{\text {th }}} h(r) \bar{F}(r) \leq \frac{\lambda}{\lambda_{\text {th }}} \mathrm{e}^{-\lambda\left|B_{1}\right| r^{d} / 2}
$$

for all large enough $r$ so that $F(r) \geq \frac{1}{2}$.

\section{Conclusions and future work}

Boolean models consisting of randomly sized spheres in $\mathbb{R}^{d}$ are long-range dependent if the sphere radii follow a power-law distribution with tail exponent $\alpha \in(d, 2 d)$. We studied secondorder statistical properties of four hard-core germ-grain models which are obtained from such 
Boolean models using natural weight-based thinning mechanisms. We found that a thinning which favors large grains preserves the power-law covariance decay of the proposed Boolean model, whereas a thinning which favors small grains does not. The power-law covariance decay is even preserved under the thinning where only isolated grains are retained (Matérn type I), and the thinning where retention is determined by independent weights (Matérn type II). The germ-grain model obtained by the Matérn type-I thinning is an interesting example of a homogeneous hard-sphere model where typical spheres have exponentially small sizes but the covariance function decays slowly according to a power law.

To keep the notation simple and the paper easy to read, the analysis carried out in this paper was restricted to spherical grains. However, we believe that this assumption can be easily relaxed to some extent following standard techniques of stochastic geometry. Another interesting open problem is to investigate how thinnings affect covariance decay properties in the light-tailed setting where the grain size distribution in the proposed Boolean model is assumed to decay rapidly.

\section{Appendix A. Regular variation}

A measurable function $f: \mathbb{R}_{+} \rightarrow \mathbb{R}$ is called regularly varying with exponent $\gamma \in \mathbb{R}$ if it is positive for all large enough input values and, for all $a>0$,

$$
\frac{f(a t)}{f(t)} \rightarrow a^{\gamma}
$$

as $t \rightarrow \infty$. A regularly varying function with exponent zero is called slowly varying. For a good overview on the theory of regular variation, see, for example, [3]. In this section we will summarize some key properties of regularly varying functions which are needed in the analysis.

Lemma A.1. Let $F$ be a distribution function on $\mathbb{R}_{+}$with a regularly varying tail of exponent $\alpha>p$. Then, for any constant $a>0$,

$$
\int_{a x}^{\infty} r^{p} F(\mathrm{~d} r) \sim\left(\frac{\alpha}{\alpha-p}\right) a^{-(\alpha-p)} \bar{F}(x) x^{p} \quad \text { as } x \rightarrow \infty .
$$

Proof. Assume first that $a=1$. The case $a \neq 1$ reduces to that because using the definition of regular variation $\bar{F}(a x)(a x)^{p} \sim a^{-(\alpha-p)} \bar{F}(x) x^{p}$. It follows by integration by parts that

$$
\int_{x}^{\infty} r^{p} F(\mathrm{~d} r)=\int_{x}^{\infty} \bar{F}(s) p s^{p-1} \mathrm{~d} s+\bar{F}(x) x^{p} .
$$

Now using Karamata's theorem [3, Theorem 1.5.11] (with $\sigma=p-1$ and $\rho=-\alpha$ ), we obtain

$$
\int_{x}^{\infty} \bar{F}(s) p s^{p-1} \mathrm{~d} s \sim-p \frac{1}{p-\alpha} x^{p} \bar{F}(x) .
$$

The claim now follows because $1-p /(p-\alpha)=\alpha /(\alpha-p)$.

Lemma A.2. Assume that $\ell$ is slowly varying. Then, for any $z_{0} \in \mathbb{R}^{d}$,

$$
\ell\left(\left|z-z_{0}\right|\right) \sim \ell(|z|) \quad \text { as }|z| \rightarrow \infty .
$$


Proof. Fix $z_{0} \in \mathbb{R}^{d}$, and write $\left|z-z_{0}\right|=a_{z}|z|$, where $a_{z}=\left|z-z_{0}\right| /|z|$. Because $a_{z} \rightarrow 1$ as $|z| \rightarrow \infty$, we can fix $m$ such that $a_{z} \in\left[\frac{1}{2}, \frac{3}{2}\right]$ for $|z|>m$. Now, for any $z \in \mathbb{R}^{d}$ such that $|z|>m$, it follows that

$$
\left|\frac{\ell\left(\left|z-z_{0}\right|\right)}{\ell(|z|)}-1\right|=\left|\frac{\ell\left(a_{z}|z|\right)}{\ell(|z|)}-1\right| \leq \sup _{a \in[1 / 2,3 / 2]}\left|\frac{\ell(a|z|)}{\ell(|z|)}-1\right| .
$$

The right-hand side above tends to 0 as $|z| \rightarrow \infty$ because $\ell(a|z|) / \ell(|z|) \rightarrow 1$ locally uniformly with respect to $a$ [3, Theorem 1.2.1].

Lemma A.3. Assume that $f$ is regularly varying with exponent $-\gamma<0$. Then there exists a constant $u>0$ such that $f(y) \leq 2 f(x)$ for all $y \geq x \geq u$.

Proof. By the uniform convergence of regularly varying functions [3, Theorem 1.5.2], $f(\lambda x) / f(x) \rightarrow \lambda^{-\gamma}$ uniformly for $\lambda \geq 1$ as $x \rightarrow \infty$. This implies that we can find $u>0$ such that $f(\lambda x) \leq 2 f(x)$ for all $x \geq u$ and all $\lambda \geq 1$. Now because $y \geq x$, we have

$$
f(y) \leq \sup _{\lambda \geq 1} f(\lambda x) \leq 2 f(x)
$$

Lemma A.4. Let $F$ be a probability measure on $\mathbb{R}_{+}$, and let $f$ and $g$ be bounded positive functions on $\mathbb{R}_{+}$such that $f(r) \sim g(r)$ as $r \rightarrow \infty$. Then

$$
\int_{r}^{\infty} f(s) F(\mathrm{~d} s) \sim \int_{r}^{\infty} g(s) F(\mathrm{~d} s) \text { as } r \rightarrow \infty .
$$

Proof. It holds that

$$
\left|1-\frac{\int_{r}^{\infty} f(s) F(\mathrm{~d} s)}{\int_{r}^{\infty} g(s) F(\mathrm{~d} s)}\right|=\left|\frac{\int_{r}^{\infty}(1-f(s) / g(s)) g(s) F(\mathrm{~d} s)}{\int_{r}^{\infty} g(s) F(\mathrm{~d} s)}\right| \leq \sup _{r \leq s}\left|1-\frac{f(s)}{g(s)}\right| .
$$

\section{Appendix B. Intersections of distant balls}

Lemma B.1. Let $F$ be a probability distribution on $\mathbb{R}_{+}$which follows a power law with tail exponent $\alpha>d$. Then, for any $r_{1}, r_{2} \geq 0$,

$$
\int_{\mathbb{R}_{+}}\left|B_{r_{1}+r}(o) \cap B_{r_{2}+r}(z)\right| F(\mathrm{~d} r) \sim c_{\alpha, d} \bar{F}(|z|)|z|^{d} \quad \text { as }|z| \rightarrow \infty,
$$

where

$$
c_{\alpha, d}=\int_{0}^{\infty}\left|B_{r}(o) \cap B_{r}\left(e_{1}\right)\right| \alpha r^{-\alpha-1} \mathrm{~d} r,
$$

and $e_{1}$ is the first unit vector in the standard basis of $\mathbb{R}^{d}$.

Proof. Because the Lebesgue measure is rotation invariant, we may assume without loss of generality that $z=u e_{1}$ for $u>0$. Fix $r_{1}, r_{2} \geq 0$, and denote the left-hand side of (B.1) by $I(u)$. We will prove the claim by first showing that

$$
I_{1}(u)=\int_{\mathbb{R}_{+}}\left|B_{r}(o) \cap B_{r}\left(u e_{1}\right)\right| F(\mathrm{~d} r) \sim c_{\alpha, d} u^{d} \bar{F}(u),
$$

and then showing that the remainder $I_{2}(u)=I(u)-I_{1}(u)$ tends to 0 faster than $u^{d} \bar{F}(u)$ as $u \rightarrow \infty$. 
To prove (B.3), let $F_{u}$ be the distribution of a random variable obtained by dividing an $F$-distributed random variable by $u$, so that $F_{u}(r)=F(u r)$. Then a change of variables shows that

$$
I_{1}(u)=u^{d} \int_{\mathbb{R}_{+}}\left|B_{r / u}(o) \cap B_{r / u}\left(e_{1}\right)\right| F(\mathrm{~d} r)=u^{d} \int_{\mathbb{R}_{+}} \phi(r) F_{u}(\mathrm{~d} r),
$$

where $\phi(r)=\left|B_{r}(0) \cap B_{r}\left(e_{1}\right)\right|$. Because $\phi$ is continuous, $r^{-d} \phi(r) \leq\left|B_{1}\right|$ for all $r>0$, and $\phi(r)=0$ for $r \leq \frac{1}{2}$, we may apply [12, Lemma 2] (with $p=d, \gamma=\alpha, q=\alpha+1$ ) to obtain

$$
\int_{\mathbb{R}_{+}} \phi(r) F_{u}(\mathrm{~d} r) \sim \bar{F}(u) \int_{0}^{\infty} \phi(r) \alpha r^{-\alpha-1} \mathrm{~d} r=c_{\alpha, d} \bar{F}(u),
$$

which implies the validity of (B.3).

To show that $I_{2}(u)$ tends to 0 faster than $u^{d} \bar{F}(u)$, note first that, for all $u>2\left(r_{1}+r_{2}\right)$,

$$
I_{2}(u)=\int_{u / 4}^{\infty} r^{d} \psi_{u}(r) F(\mathrm{~d} r) \leq\left(\sup _{r>u / 4} \psi_{u}(r)\right) \int_{u / 4}^{\infty} r^{d} F(\mathrm{~d} r),
$$

where

$$
\psi_{u}(r)=\left|B_{r_{1} / r+1}(o) \cap B_{r_{2} / r+1}\left(\frac{e_{1} u}{r}\right)\right|-\left|B_{1}(o) \cap B_{1}\left(\frac{e_{1} u}{r}\right)\right| .
$$

The equality in (B.4) follows because $\psi_{u}(r)=0$ when $u>2\left(r_{1}+r_{2}\right)$ and $r<u / 4$.

Note that, by Lemma A.1, the integral on the right-hand side of (B.4) is asymptotically equivalent to

$$
\int_{u / 4}^{\infty} r^{d} F(\mathrm{~d} r) \sim \frac{\alpha}{\alpha-d} 4^{\alpha-d} \bar{F}(u) u^{d} .
$$

In light of (B.4), it hence suffices to show that

$$
\sup _{r>u / 4} \psi_{u}(r) \rightarrow 0
$$

as $u \rightarrow \infty$. This will be done by inspecting the geometry of $\psi_{u}$. Because the intersection of the unit balls above is a subset of the intersection of the larger balls, we can bound the nonnegative function $\psi_{u}$ using the annuli around the unit balls, so that

$$
\begin{aligned}
\psi_{u}(r) & =\left|\left(B_{r_{1} / r+1}(o) \cap B_{r_{2} / r+1}\left(\frac{e_{1} u}{r}\right)\right) \backslash\left(B_{1}(o) \cap B_{1}\left(\frac{e_{1} u}{r}\right)\right)\right| \\
& \leq\left|B_{r_{1} / r+1}(o) \backslash B_{1}(o)\right|+\left|B_{r_{2} / r+1}\left(\frac{e_{1} u}{r}\right) \backslash B_{1}\left(\frac{e_{1} u}{r}\right)\right| \\
& =\left|B_{1}\right|\left(\left(\frac{r_{1}}{r+1}\right)^{d}-1\right)+\left|B_{1}\right|\left(\left(\frac{r_{2}}{r+1}\right)^{d}-1\right) \\
& \leq 2\left|B_{1}\right|\left(\left(\frac{r_{1} \vee r_{2}}{r}+1\right)^{d}-1\right) .
\end{aligned}
$$

Because this bound is valid for all $u$, we conclude (B.5), and the proof is complete.

Lemma B.2. Let $F$ be a probability distribution on $\mathbb{R}_{+}$which follows a power law with tail exponent $\alpha>d$. Then there exist constants $u>0$ and $c>0$ such that

$$
\int_{\mathbb{R}_{+}}\left|B_{r_{1}+r}(o) \cap B_{r_{2}+r}(z)\right| F(\mathrm{~d} r) \leq c \bar{F}(|z|)|z|^{d}
$$

whenever $|z|>u$ and $r_{1}+r_{2} \leq|z| / 2$. 
Proof. Observe first using Lemma A.1 that

$$
\int_{|z| / 4}^{\infty} r^{d} F(\mathrm{~d} r) \sim c_{1}|z|^{d} \bar{F}(|z|)
$$

where $c_{1}=4^{\alpha-d} \alpha /(\alpha-d)$. Hence, we may fix a constant $u>0$ such that

$$
\int_{|z| / 4}^{\infty} r^{d} F(\mathrm{~d} r) \leq 2 c_{1}|z|^{d} \bar{F}(|z|)
$$

whenever $|z|>u$.

Assume now that $|z|>u$ and $r_{1}+r_{2} \leq|z| / 2$. In this case the intersection on the left-hand side of (B.6) is nonempty only when $r>|z| / 4$. For any such $r>|z| / 4$, a crude estimate shows that

$$
\left|B_{r_{1}+r}(o) \cap B_{r_{2}+r}(z)\right| \leq\left|B_{r_{1}+r}(o)\right| \leq\left|B_{1}\right|\left(r_{1}+r_{2}+r\right)^{d},
$$

which together with the inequality $r_{1}+r_{2} \leq|z| / 2<2 r$ shows that

$$
\left|B_{r_{1}+r}(o) \cap B_{r_{2}+r}(z)\right| \leq 3^{d}\left|B_{1}\right| r^{d} .
$$

As a consequence,

$$
\int_{\mathbb{R}_{+}}\left|B_{r_{1}+r}(o) \cap B_{r_{2}+r}(z)\right| F(\mathrm{~d} r) \leq 3^{d}\left|B_{1}\right| \int_{|z| / 4}^{\infty} r^{d} F(\mathrm{~d} r),
$$

so that, by virtue of (B.7), the claim holds for $c=2 c_{1} 3^{d}\left|B_{1}\right|$.

Lemma B.3. Let $F$ be a probability distribution on $\mathbb{R}_{+}$which follows a power law with tail exponent $\alpha>d$, and let $h$ be an arbitrary positive function. Then there exist constants $u>0$ and $c>0$ such that

$$
\left(|z|^{d} \bar{F}(|z|)\right)^{-1} \int_{\mathbb{R}_{+}}\left|B_{r}(o) \cap B_{r}(z)\right| h(r) F(\mathrm{~d} r) \leq c \sup _{r \geq|z| / 2} h(r)
$$

for $|z|>u$.

Proof. By using Lemma B.2, fixing the constants $u$ and $c$ as in the lemma, and noting that the integrand vanishes for $r \leq|z| / 2$, we see that

$$
\int_{\mathbb{R}_{+}}\left|B_{r}(o) \cap B_{r}(z)\right| h(r) F(\mathrm{~d} r) \leq h^{*}(z) \int_{\mathbb{R}_{+}}\left|B_{r}(o) \cap B_{r}(z)\right| F(\mathrm{~d} r) \leq c h^{*}(z) \bar{F}(|z|)|z|^{d}
$$

for all $|z|>u$, where $h^{*}(z)=\sup _{r \geq|z| / 2} h(r)$.

\section{Acknowledgements}

We thank Volker Schmidt for suggesting to study this type of problem a long time ago back in 2005. We thank Antti Penttinen for inspiring discussions and valuable comments, and the anonymous referees for helpful suggestions on the presentation. These results were presented at the eighth World Congress in Probability and Statistics, Istanbul, 2012. Mikko Kuronen was supported by the Finnish Doctoral Programme in Stochastics and Statistics. 


\section{References}

[1] Andersson, J., Häggström, O. and Månsson, M. (2006). The volume fraction of a non-overlapping germgrain model. Electron. Commun. Prob. 11, 78-88.

[2] Baccelli, F. and BŁaszczyszyn, B. (2009). Stochastic Geometry and Wireless Networks, Vol. II. NoW Publishers. Available at http://hal.inria.fr/inria-00403040.

[3] Bingham, N. H., Goldie, C. M. and Teugels, J. L. (1987). Regular Variation. Cambridge University Press.

[4] Böнm, S. And Schmidt, V. (2003). Palm representation and approximation of the covariance of random closed sets. Adv. Appl. Prob. 35, 295-302.

[5] Clauset, A., Shalizi, C. R. and Newman, M. E. J. (2009). Power-law distributions in empirical data. SiaM Rev. 51, 661-703.

[6] Daley, D. J. (1999). The Hurst index of long-range dependent renewal processes. Ann. Prob. 27, $2035-2041$.

[7] Daley, D. J. And Vere-Jones, D. (2008). An Introduction to the Theory of Point Processes, Vol. II, 2nd edn. Springer, New York.

[8] Daley, D. J. and Vesilo, R. (1997). Long range dependence of point processes, with queueing examples. Stoch. Process. Appl. 70, 265-282.

[9] Demichel, Y., Estrade, A., Kratz, M. and Samorodnitsky, G. (2011). How fast can the chord length distribution decay? Adv. Appl. Prob. 43, 504-523.

[10] HaengGi, M. (2011). Mean interference in hard-core wireless networks. IEEE Commun. Lett. 15, $792-794$.

[11] Jones, B. J. T., Martínez, V. J., SaAr, E. and Trimble, V. (2005). Scaling laws in the distribution of galaxies. Rev. Modern Phys. 76, 1211-1266.

[12] Kaj, I., Leskelä, L., Norros, I. and Schmidt, V. (2007). Scaling limits for random fields with long-range dependence. Ann. Prob. 35, 528-550.

[13] Kulik, R. AND Szekli, R. (2001). Sufficient conditions for long-range count dependence of stationary point processes on the real line. J. Appl. Prob. 38, 570-581.

[14] Månsson, M. and Rudemo, M. (2002). Random patterns of nonoverlapping convex grains. Adv. Appl. Prob. 34, 718-738.

[15] Matérn, B. (1960). Spatial Variation: Stochastic Models and Their Application to Some Problems in Forest Surveys and Other Sampling Investigations. Meddelanden Fran Statens Skogsforskningsinstitut, Stockholm.

[16] Molchanov, I. (2005). Theory of Random Sets. Springer, London.

[17] Nguyen, T. V. and Baccelli, F. (2012). Generating functionals of random packing point processes: from hard-core to carrier sensing. Preprint. Available at http://arxiv.org/abs/1202.0225v2.

[18] OHSER, J. AND Mücklich, F. (2000). Statistical Analysis of Microstructures in Materials Science. John Wiley.

[19] Samorodnitsky, G. (2006). Long range dependence. Found. Trends Stoch. Systems 1, 163-257.

[20] Schneider, R. AND WeIL, W. (2008). Stochastic and Integral Geometry. Springer, Berlin.

[21] Schüth, F., Sing, K. S. W. And Weitkamp, J. (eds) (2002). Handbook of Porous Solids. Wiley-VCH.

[22] Snethlage, M., Martínez, V. J., Stoyan, D. And SaAr, E. (2002). Point field models for the galaxy point pattern. Astron. Astrophys. 388, 758-765.

[23] Stoyan, D., Kendall, W. S. and Mecke, J. (1995). Stochastic Geometry and Its Applications, 2nd edn. John Wiley, Chichester.

[24] Vamvakos, S. And Anantharam, V. (1998). On the departure process of a leaky bucket system with long-range dependent input traffic. Queueing Systems 28, 191-214. 